\title{
Inventio, dispositio, elocutio: tre lenti per l'analisi di argomentazioni nei libri di testo di geometria
}

\section{Inventio, dispositio, elocutio: three lenses for analysing argumentation in geometry textbooks}

\section{Michele Canducci ${ }^{\bullet}$, Andrea Rocci ${ }^{\bullet}$ e Silvia Sbaragli ${ }^{\bullet}$}

- Dipartimento formazione e apprendimento, SUPSI - Locarno, Svizzera

- Facoltà di comunicazione, cultura e società, USI - Lugano, Svizzera

凶 michele.canducci@supsi.ch, andrea.rocci@usi.ch, silvia.sbaragli@supsi.ch

Sunto / A partire dal corpus del progetto Italmatica. Comprendere la matematica a scuola, fra lingua comune e linguaggio specialistico del Fondo nazionale svizzero, viene presentata un'analisi di esempi tratti dai libri di testo di geometria in lingua italiana della scuola primaria e secondaria di primo grado. L'analisi si basa sull'applicazione delle categorie di tipo retorico classico: inventio, dispositio ed elocutio, oggi afferenti ai domini degli studi linguistici, in particolare delle teorie dell'argomentazione. Attraverso l'analisi condotta, vengono evidenziate da un lato la profondità delle riflessioni che queste lenti teoriche consentono di raggiungere nello sviscerare un testo argomentativo di matematica, dall'altro la grande varietà di scelte possibili adottate dai libri di testo, che possono avere un effetto comunicativo sul lettore-studente.

Parole chiave: inventio; dispositio; elocutio; argomentazioni; libri di testo di geometria.
Abstract / Starting from the corpus of the project Italmatica. Comprendere la matematica a scuola, fra lingua comune e linguaggio specialistico of the Swiss National Science Foundation, an analysis of examples from Italian-language geometry textbooks of primary and secondary schools is presented. The analysis is based on the application of classical rhetorical categories: inventio, dispositio and elocutio, which are nowadays related to the domains of linguistic studies, in particular theories of argumentation. Through the analysis, we highlight on one hand the depth of the reflections that these theoretical lenses allow us to reach in dissecting an argumentative text in mathematics, on the other hand, we show the great variety of possible choices adopted by textbooks, which can have a communicative effect on the reader-student.

Keywords: inventio; dispositio; elocutio; argumentation; geometry textbooks.

(C) 2021 Canducci Michele, Rocci Andrea e Sbaragli Silvia. Questo è un articolo Open Access, sottoposto a un processo di revisione tra pari a doppio cieco, pubblicato dal Centro competenze didattica della matematica e dal Servizio risorse didattiche e scientifiche, eventi e comunicazione del DFA-SUPSI in collaborazione con il DECS. L'articolo è distribuito sotto i termini della Licenza Creative Commons Attribuzione 4.0 Internazionale (CC BY 4.0) che permette di usare, condividere e modificare l'articolo su qualsiasi mezzo a patto che I'autore e la fonte originale siano citati. 
«Physica ista ipsa et mathematica et quae paulo ante ceterarum
artium propria posuisti, scientiae sunt eorum, qui illa
profitentur; illustrare autem oratione si quis istas ipsas artes velit,
ad oratoris ei confugiendum est facultatem».
(Cicerone, De oratore, l, 61)

«È chiaro che la fisica, la matematica o le altre scienze sono proprie di coloro che le professano, ma se vogliamo che esse siano illustrate in un discorso chiaro ed efficace, bisogna rivolgersi all'abilità specifica dell'oratore».

(Cicerone, De oratore, I, 61)

Questo contributo si inserisce all'interno del progetto Italmatica. Comprendere la matematica a scuola, fra lingua comune e linguaggio specialistico. ${ }^{1}$ II progetto ha come obiettivo I'individuazione, la raccolta e l'analisi, dal punto di vista linguistico e matematico, di un corpus di libri di testo scolastici di matematica in lingua italiana della scuola primaria ${ }^{2}$ e secondaria di primo grado, ${ }^{3}$ al fine di delinearne le caratteristiche e i possibili ostacoli per la comprensione degli alunni. Si è concentrata l'attenzione sull'ambito geometrico; in particolare, il tema indagato è quello dei poligoni. Poiché tale argomento viene proposto con continuità dalla seconda primaria alla terza secondaria di primo grado, in accordo con l'idea di percorso a spirale per la costruzione di competenze matematiche, abbiamo potuto contare su un bacino di libri di testo riferito a sette anni di scolarità. In questo modo si è composto un corpus, denominato DFA-Italmatica, ${ }^{4}$ sul quale è stato possibile effettuare analisi a diversi livelli, focalizzate su aspetti differenti e facenti uso di una grande varietà di approcci qualitativi e quantitativi (Sbaragli \& Demartini, 2021). Queste analisi sono state condotte da un team interdisciplinare di ricercatori in didattica della matematica e in linguistica, e hanno messo in luce un panorama complesso, dal quale emerge chiaramente come le diverse scelte di tipo linguistico e testuale, effettuate dai costruttori di senso di un libro di testo (Bezemer \& Kress, 2010), possono influenzare il lettore in fase di apprendimento della matematica. ${ }^{5}$

In questo contributo intendiamo condurre un ulteriore passo verso una visione sempre più integrata fra la dimensione linguistica e l'apprendimento della matematica, proponendo l'analisi di argomentazioni realizzata attraverso le categorie retoriche dell'inventio, dispositio ed elocutio.

In particolare, ci si chiede se la lettura delle porzioni argomentative presenti nei libri di testo tramite queste categorie di analisi possa mettere in luce aspetti significativi per la didattica della matematica, che tipicamente non riescono a emergere attraverso altri tipi di analisi condotte abitualmente in que-

\footnotetext{
1. Progetto 176339 del Fondo nazionale svizzero per la ricerca scientifica.

2. La scuola primaria in Italia dura cinque anni e corrisponde alla scuola elementare nel Canton Ticino.

3. La scuola secondaria di primo grado in Italia dura tre anni e corrisponde ai primi tre anni di scuola media nel Canton Ticino.

4. I testi del corpus sono stati raccolti tra quelli editi in Italia e nei cantoni italofoni della Svizzera (Canton Ticino e Canton Grigioni), arrivando a comporre un corpus di 142 titoli. Tra questi, 129 provengono dal variegato e ampio contesto editoriale italiano; 13 libri dal contesto svizzero, di cui 7 provengono dal Canton Ticino e 6 dal Canton Grigioni. La minore presenza di libri di testo svizzeri è dovuta al fatto che, soprattutto in Canton Ticino, I'utilizzo del libro di testo in ambito didattico non è diffuso. Per un approfondimento dei criteri con i quali è stato costruito il corpus, si veda Sbaragli e Demartini (2021).

5. Tra le tematiche trattate citiamo: gli aspetti strutturali di architettura testuale dei manuali (Demartini, Sbaragli \& A. Ferrari, 2020); gli aspetti lessicali e morfosintattici dei manuali (Canducci, 2020; Canducci et al., 2019a, 2019b; Canducci, Demartini \& Sbaragli, 2021; Demartini, Fornara \& Sbaragli, 2020; Demartini \& Sbaragli, 2019); gli aspetti legati al rapporto multimodale fra testo e figure nei manuali (Canducci, 2019; Canducci, Rocci \& Sbaragli, 2021); gli aspetti legati alle diverse modalità con le quali vengono proposti movimenti testuali di tipo argomentativo (Sbaragli, Canducci \& Demartini, 2021).
} 
sto ambito di ricerca. Nel fare questo, proporremo dunque un quadro teorico derivante dal mondo della linguistica, in particolare riferito a studi di teoria dell'argomentazione, con il fine da un lato di poter dare un contributo nuovo e originale all'attuale e ampia ricerca che viene fatta oggi in didattica della matematica sul tema dell'argomentazione ${ }^{6}{ }^{6}$ dall'altro di rendere conto della grande varietà di scelte comunicative che possono essere effettuate nei libri di testo, e che potrebbero avere un certo effetto sui lettori-studenti.

Per affrontare questo compito, esporremo dapprima i motivi che ci hanno condotto a considerare alcune porzioni di libri di testo come argomentazioni, calandoli all'interno di un caso di studio (par. 2); chiariremo poi alcuni aspetti relativi alle categorie di analisi che utilizzeremo (par. 3), focalizzandoci sugli elementi che saranno utili per analizzare le porzioni argomentative dei libri di testo di matematica; analizzeremo poi in profondità tramite queste lenti di analisi il caso di studio (par. 4) per metterlo a confronto con altre scelte presenti nei libri di testo del corpus DFA-Italmatica (par. 5), arrivando così a trarre alcune considerazioni.

\section{Un caso di studio: la somma delle ampiezze degli angoli interni di un poligono}

Presentiamo una porzione di un libro di testo italiano di matematica rivolto a studenti di prima secondaria di primo grado nel quale si accompagna il lettore a riconoscere che la somma delle ampiezze degli angoli interni di un poligono di $n$ lati è pari a $(n-2) \cdot 180^{\circ}$ (Figura 1 ).

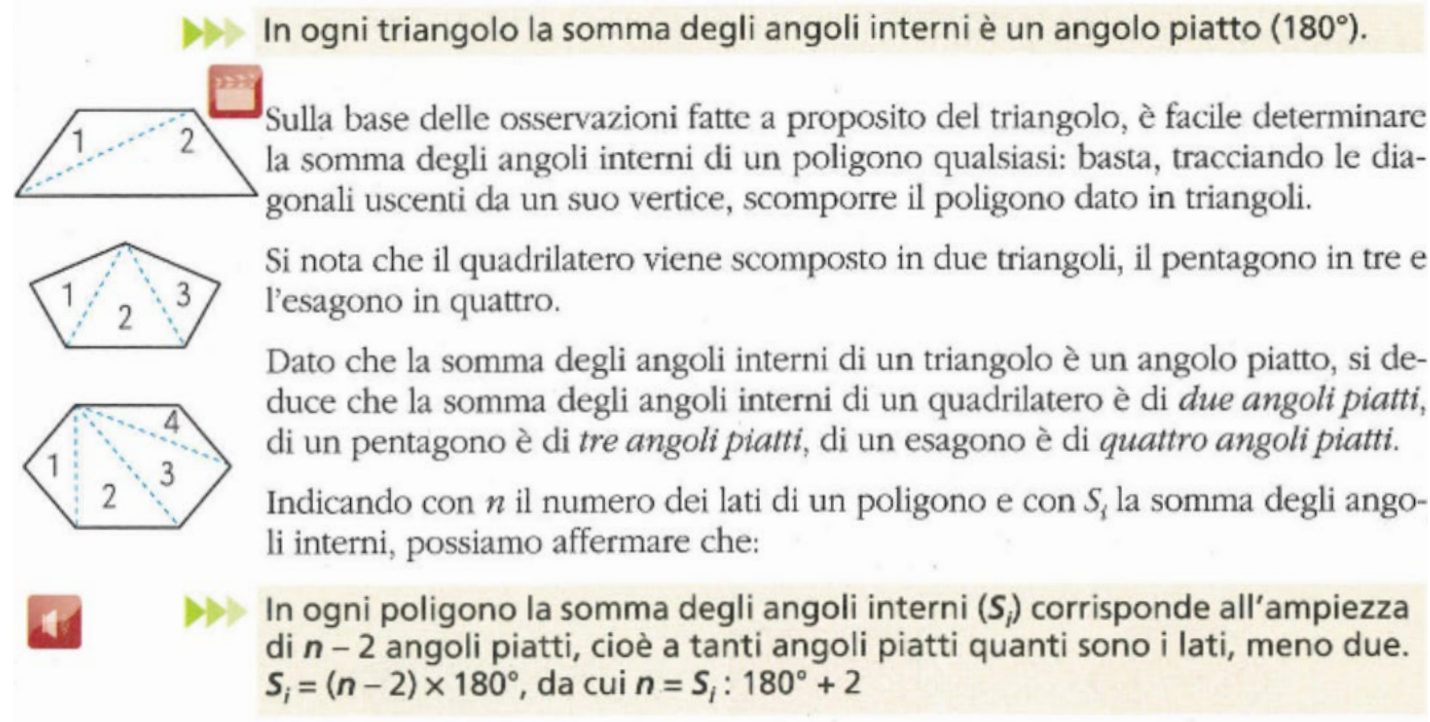

Figura 1. Porzione di libro di testo italiano di prima scuola secondaria di primo grado (libro 2 _6 del corpus, p. 176).

Dal punto di vista matematico, la strategia con la quale si giunge al risultato sfrutta l'idea di scomporre un poligono generico di $n$ lati in $n-2$ triangoli attraverso una triangolazione che consiste nel

6. Non approfondiremo in questo contributo il tema dell'argomentazione trattato nell'ambito della ricerca in didattica della matematica, in quanto focalizzato prevalentemente su un discorso che si sviluppa nella pratica didattica matematica delle classi, mentre qui ci occupiamo di porzioni testuali presenti nei libri di testo di matematica, rigidi dal punto di vista dell'interazione comunicativa con lo studente-lettore. Tuttavia queste interessanti discussioni proseguono da decenni e hanno prodotto una vasta letteratura a cui rimandiamo (si veda ad esempio http://www.lettredelapreuve.org/). 
tracciare tutte le diagonali che hanno come estremo un vertice fissato. Sapendo poi che la somma degli angoli interni di un triangolo è pari all'ampiezza di un angolo piatto, si inferisce che la somma degli angoli interni di un poligono di $n$ lati è pari a $n-2$ angoli piatti.

Ci domandiamo: di che tipo di discorso si tratta? Può essere considerata una dimostrazione matematica? Pur avendo assunto nel corso dei secoli forme diverse, le dimostrazioni matematiche posseggono una comune struttura che può essere riassunta con le parole di Balacheff:

«Una dimostrazione è una successione di enunciati tale che ciascun enunciato o è un'ipotesi, o è un enunciato la cui validità è addirittura già stabilita (teorema), o ammessa (assioma), o è dedotto da enunciati che lo precedono secondo una regola esplicita e condivisa».?

(Balacheff, 2001, p. 4)

Seguendo questa definizione, la successione di enunciati presenti nell'esempio non può essere considerata una dimostrazione, perché se da un lato è evidente che in questa porzione di testo sia presentato un discorso che dà delle ragioni a sostegno della proposizione finale, dall'altro bisogna ammettere che si utilizzano inferenze di tipo diverso: alcune di queste sono di tipo deduttivo, come nel caso del passaggio nel terzo blocco testuale introdotto dal "dato che"; invece, lo schema globale del ragionamento proposto nello stralcio di testo segue un altro tipo di inferenza, solitamente chiamata con il termine induzione. ${ }^{8}$ Questo metodo consiste nell'inferire dal particolare al generale: dopo aver verificato che una certa proprietà è valida per un certo numero di elementi appartenenti a una classe, si afferma che tale proprietà è valida per la totalità degli elementi della classe. ${ }^{9} \mathrm{Nel}$ nostro caso di studio, appare evidente l'utilizzo di tale metodo: in tre casi di poligoni vale la proprietà che la somma delle ampiezze dei loro angoli interni corrisponde all'ampiezza di tanti angoli piatti quanti sono il numero dei lati del poligono considerato diminuito di 2 (che corrisponde al numero di triangoli che si ottengono secondo la logica di triangolazione scelta); da ciò si inferisce induttivamente che tale proprietà vale per tutti i poligoni.

Ora, se si considera che, in generale, un'argomentazione può essere definita come un «processo che "aiuta" I'interlocutore a riconoscere qualcosa fornendo (direttamente o indirettamente) una opportuna giustificazione» (Rigotti \& Greco, 2009, p. 4, traduzione degli autori), essa può essere interpretata dal punto di vista epistemologico come iperonimo di dimostrazione: ${ }^{10}$ in altre parole, l'insieme delle dimostrazioni rappresenta un sottoinsieme dell'insieme delle argomentazioni, il quale sarebbe dunque formato dai due sottoinsiemi delle argomentazioni dimostrative e delle argomentazioni non dimostrative. Se nelle argomentazioni dimostrative possono comparire solo passaggi deduttivi, nelle argomentazioni non dimostrative possono comparire processi inferenziali diversi, quali, ad esempio, quelli di tipo induttivo (o abduttivo).

In definitiva, possiamo interpretare la porzione di testo qui presentata come argomentazione, in particolare come argomentazione non dimostrativa, sviluppata attraverso passaggi inferenziali non solo deduttivi, tenendo anche conto che l'intento di ogni argomentazione è in generale di convincere un uditorio riguardo alla plausibilità di una certa tesi. Da questo punto di vista, ci sembra infatti che, laddove i limiti dovuti ad esempio (ma non solo) alla giovane età dei discenti non consentano un approccio pedagogico di tipo esclusivamente dimostrativo ai risultati matematici, tra gli intenti di

7. Questo approccio alla dimostrazione non è I'unico. Ad esempio, nell'approccio di Tarski, basato sulla semantica, un enunciato segue dai precedenti se è soddisfatto in tutte le interpretazioni che soddisfano i medesimi (e non in base a una o più regole). Per un approfondimento di questa impostazione, e delle possibili conseguenze sul piano logico e filosofico, si veda Novaes (2020).

8. Da non confondere con il principio di induzione matematica, che è invece un assioma dell'aritmetica di Peano con il quale si possono dimostrare, ad esempio, numerosi teoremi di aritmetica elementare.

9. Ovviamente, questo procedimento non dà la certezza che l'affermazione finale sia vera: al più si può concludere come sia verosimile che la suddetta proprietà valga nella totalità dei casi in oggetto.

10. II termine "iperonimo" indica un'unità lessicale di significato più generico ed esteso rispetto a una o più unità lessicali che sono in essa incluse. Ad esempio, "fiore" è un iperonimo di "garofano". 
un libro di testo di matematica per questi livelli scolastici possa (anzi forse debba) rientrare anche il convincere ${ }^{11}$ il lettore riguardo alla plausibilità di alcuni fatti matematici, tramite argomentazioni più o meno dimostrative.

Fatta questa premessa, rivolgiamo ora l'attenzione al chiarire alcuni aspetti teorici relativi alle categorie di analisi che utilizzeremo. Nel farlo, ci porremo in una prospettiva contemporaneamente diacronica e sincronica, nella quale intrecceremo le riflessioni degli studi classici di retorica con quelle relative ai moderni studi della teoria dell'argomentazione e di altre discipline del linguaggio.

\title{
3 Largomentazione come discorso di ragione e persuasione
}

Dal punto di vista dei contenuti e delle problematiche a cui si rivolge, la teoria dell'argomentazione è una disciplina antica, che trae origine nella pratica sofistica, nella dialettica socratico-platonica, nell'opera di Aristotele, e in seguito negli studi retorici dell'epoca romana. Imprescindibile per gli sviluppi fino alla modernità è la distinzione tra dialettica e retorica, operata principalmente da Aristotele: la dialettica si occupa del ragionamento attorno al verosimile, ${ }^{12}$ di tesi cui per forza di cose si può solo aderire con intensità variabile, ed è concepita dallo stesso Aristotele «come l'arte di ragionare partendo da opinioni generalmente accettate» (Perelman \& Olbrechts-Tyteca, 1958/2013, p. 7); la retorica, complementare alla dialettica, ha come oggetto il discorso persuasivo nei confronti di un uditorio, e insegna

\begin{abstract}
«Come si deve dire qualcosa - secondo quali schemi, seguendo quali criteri, con quali cautele - perché ciò che viene detto risulti un mezzo efficace per consentire il fine del nostro dire-fare, cioè la produzione di determinati effetti sull'uditore (convincerlo circa la credibilità di un'opinione, indurlo a compiere o ad astenersi dal compiere una data azione, portarlo a modificare certi suoi atteggiamenti, sentimenti ecc.)».
\end{abstract}

(Cattani, 1994, p. 166)

Si semplifica la questione, ma non si sbaglia di molto, affermando dunque che se la dialettica è maggiormente focalizzata sugli aspetti di ragionevolezza del discorso, la retorica è più spostata sull'efficacia persuasiva dello stesso. Questa caratterizzazione è sostanzialmente rimasta tale per duemila anni, fino all'avvento di quella che oggi è conosciuta come teoria dell'argomentazione, espressione con la quale si indica un filone di riflessioni nato a metà del XX secolo, e che si distingue dagli studi classici principalmente per il tentativo di guardare all'argomentazione da un punto di vista organico, nel quale convergono tanto la tradizione retorica quanto quella dialettica. ${ }^{13}$ Ecco il perché dell'espressione La nuova retorica, sottotitolo del celebre Trattato dell'argomentazione del 1958 di Perelman e Olbrechts-Tyteca: con questo titolo gli autori volevano intendere che ogni argomentazione, anche quella apparentemente più impersonale, è di tipo retorico, perché si sviluppa in funzione di un effetto sulla realtà (Perelman \& Olbrechts-Tyteca, 1958/2013, p. 8). Con questo approccio è possibile guardare alle pratiche e ai discorsi argomentativi attraverso una lente pragmatica (van Eemeren, 2015)

11. In generale, e soprattutto per livelli scolastici più alti rispetto a quelli qui considerati, l'intento di un libro di testo a uso didattico sembra essere maggiormente legato, più che al convincere, al mettere in luce legami fra proprietà matematiche diverse, o illustrare schemi di ragionamento tipici di un'area tematica. Questo nell'ottica in cui l'esplicitazione delle relazioni di coerenza, relazioni che l'uditorio a cui ci si riferisce potrà valutare in modo autonomo, costituisca il punto fondamentale di ogni argomentazione (Mercier \& Sperber, 2017).

12. La dialettica è quindi diversa dall'analitica, nella quale si mettono in luce i meccanismi della deduzione che parte da premesse vere per giungere a conclusioni logicamente fondate e necessariamente vere.

13. Ricostruire storicamente i passaggi, tutt'altro che banali, che hanno portato dalla visione tradizionale legata alla dialettica e retorica classica alla moderna teoria dell'argomentazione, è un'impresa che esula dagli scopi di questo contributo. Per chi avesse intenzione di approfondire, rimandiamo a van Eemeren (2013) e Rigotti e Greco (2019). 
che accompagna alla imprescindibile dimensione di ragionevolezza quella di efficacia persuasiva, arrivando a parlare di «ragionevolezza dell'impegno persuasivo» (Rocci, 2017), cioè di una posizione in cui si recupera «l'idea che la persuasività sia un reale contributo alla ragionevolezza, sia necessaria ad una comunicazione pienamente ragionevole», in cui chi argomenta «non ritiene solo che sia possibile essere ragionevoli e persuasivi, ma anche che non sia possibile essere pienamente ragionevoli senza un impegno persuasivo, un impegno ad aver cura delle circostanze che favoriscono nel mio interlocutore l'uso della ragione» (Rocci, 2017, pp. 102-103).

In altre parole, chi argomenta, sia esso un politico, un giornalista, un avvocato, un insegnante o, come nel nostro caso, un libro di testo di geometria, dovrebbe saper utilizzare gli strumenti retorici non per manipolare, ma per rendere la propria argomentazione davvero pragmaticamente ragionevole, cioè passibile di essere accolta pienamente da un interlocutore invitato a coglierne la ratio. I moderni studi di comunicazione e di argomentazione si focalizzano proprio su questo punto, cioè sul rapporto che c'è tra la dimensione di correttezza dialettica ${ }^{14}$ e la dimensione dell'efficacia comunicativa di un discorso. È in questa delicata sinergia che entrano in gioco alcune delle categorie della retorica classica e moderna quali sono l'inventio, la dispositio e l'elocutio, delle quali parleremo, per forza di cose in modo esplorativo, non enciclopedico, ${ }^{15}$ nel prossimo paragrafo, cercando poi di applicarle alle porzioni di libri di testo di geometria.

\title{
3.1 Inventio, dispositio, elocutio
}

La tradizione retorica latina, ${ }^{16}$ che molto deve agli studi retorici greci precedenti, suddivide l'arte dei discorsi oratori in cinque parti: inventio, dispositio, elocutio, memoria e actio. Se ancora oggi, nei nuovi studi di retorica, si utilizzano sostanzialmente queste etichette applicate all'organizzazione del discorso argomentativo e ai suoi componenti su vari livelli (tematico, stilistico, sintattico, prosodico ecc.), resistendo dunque all'indebolimento delle impalcature su cui poggiava l'intero sistema classificatorio, è perché «evidentemente tali etichette servono ancora a designare fatti che oggi si analizzano con strumenti del tutto diversi da quelli che un tempo erano serviti per la costruzione delle impalcature» (Mortara Garavelli, 1988/2020, p. 80).

Nella Rhetorica ad Herennium le cinque sezioni dell'arte del dire sono definite come segue:

\author{
«L'invenzione [inventio] è la capacità di trovare argomenti veri o verosimili che rendano la causa \\ convincente. \\ La disposizione [dispositio] è l'ordinamento e la distribuzione degli argomenti; essa indica il luogo \\ che ciascuno di essi deve occupare. \\ L'eloquio [elocutio] è l'uso delle parole e delle frasi opportune in modo da adattarsi all'inventio. \\ La memoria [memoria] è la tenace presenza nel pensiero degli argomenti, delle parole e della loro \\ disposizione. \\ La dizione [actio] è la capacità di regolare in modo gradito la voce, I'aspetto, il gesto».
}

(Cornificio, Rhetorica ad Herennium, I, 2, 3)

Nel momento in cui il discorso non è presentato nella variazione diamesica dell'oralità, bensì dello scritto, le sezioni della retorica si riducono sostanzialmente alle prime tre, perché la memoria e l'actio «riguardano l'esecuzione orale di discorsi scritti per essere recitati (memorizzati o anche letti)» (Mortara Garavelli, 1988/2020, p. 84), e vengono dunque trascurate nelle trattazioni dedicate alla com-

\footnotetext{
14. La correttezza dialettica di un'argomentazione si sviluppa sul piano logico, ovviamente, ma anche extralogico, se si pensa ad esempio al fatto che alcuni argomenti possono essere ammessi o non ammessi a seconda del contesto in cui vengono presentati.

15. Per chi intendesse approfondire questi aspetti consigliamo Mortara Garavelli (1988/2020).

16. Questa tradizione si rifà principalmente all'opera di Cicerone (il De Inventione e il De Oratore), Cornificio (Rethorica ad Herennium) e Quintiliano (Institutio Oratoria)
} 
posizione scritta. In termini attuali, gli ambiti di interesse delle tre categorie dell'inventio, dispositio ed elocutio non si discostano molto da quelli individuati dagli antichi. Nei prossimi paragrafi cercheremo di approfondirle, individuandone le caratteristiche utili ai nostri scopi e fornendo, laddove sarà opportuno, alcuni esempi di declinazione in ambito matematico.

\subsubsection{L'inventio: il reperimento degli argomenti}

L'inventio riguarda la progettazione del discorso persuasivo: si occupa cioè di reperire e scegliere tipologie di prove e argomenti che rendano convincente la tesi cui si vuol giungere.

Da un punto di vista tanto classico quanto moderno, in questa sezione si affrontano i tipi di ragionamento e la tematica dei tòpoi (loci in latino, luoghi in italiano). Nella sistemazione aristotelica dell'inventio, ci si sofferma in particolare sulle strutture tipiche dell'argomentazione quali sono l'esempio e l'entimema.

Per Aristotele, l'esempio «rappresenta l'analogo retorico dell'induzione [...] poiché consiste nel dimostrare, sulla base di molti casi simili, che le cose stanno in un certo modo» (Piazza, 2015, p. 115). Nella rielaborazione moderna dell'esempio, Perelman e Olbrechts-Tyteca (1958/2013) lo considerano come uno dei tre tipi di argomenti che si basano sul "caso particolare", insieme all'illustrazione e al modello: la descrizione di un fatto è un esempio quando serve a dare fondamento a una regola; assume il carattere di un'illustrazione quando rafforza «l'adesione a una regola riconosciuta e ammessa, fornendo dei casi particolari che chiariscono l'enunciato generale» (Perelman \& Olbrechs-Tyteca, 1958/2013, p. 389); il modello, invece, corrisponde all'esempio e all'illustrazione nell'ambito dell'agire pratico, essendo «l'insieme dei comportamenti (o degli attributi di un ente qualsiasi) su cui si può fondare o coi quali si può illustrare una regola generale di condotta» (Mortara Garavelli, 1988/2020, p. 108). Materia dell'inventio riferita al "caso particolare" è, com'è naturale, anche la scelta di quali e quanti esempi proporre all'uditorio: infatti da un lato si tratterà di scegliere la tipologia di esempio più opportuna in funzione dell'uditorio cui ci si riferisce, dall'altro di decidere quanti esempi proporre a sostegno di una tesi, non dimenticando che dal punto di vista della retorica classica «il giusto mezzo tra i due opposti eccessi, che sono il dire troppo o troppo poco, si otterrà con l'esporre "quanto bisogna" e "quanto basta": il necessario e sufficiente» (Mortara Garavelli, 1988/2020, p. 95), e questo vale in generale per tutte le parti del discorso persuasivo.

Per quanto riguarda l'entimema, invece, Aristotele lo definisce in questo modo: «esso è quando, date certe premesse, risulta per mezzo di esse qualcosa di altro e di ulteriore per il fatto che esse sono tali o universalmente o per lo più» (Aristotele, Retorica, I, 2, 1356b). L'entimema è l'analogo retorico del sillogismo logico: se quest'ultimo, partendo da premesse necessariamente vere, dà come risultato una verità inconfutabile, l'entimema giunge a conclusioni probabili e confutabili, poiché si basa su premesse verosimili, ma non necessariamente vere. Così come i sillogismi, gli entimemi sono strutturati secondo la terna premessa maggiore, premessa minore e tesi. Ora, generalmente, quando si vuole convincere circa la validità di un'affermazione, l'indagine volta al reperimento delle proposizioni già accettate $o$ accettabili per I'uditorio, che possano essere pertinentemente invocate come premesse, costituisce il cuore dell'inventio. Per facilitare questo sforzo, per aiutare cioè chi deve argomentare nella ricerca di premesse utili a sostenere la tesi conclusiva, tutti gli studi retorici da Aristotele in poi hanno cercato di raggruppare, suddividendolo in categorie, il materiale utile poter trovare più facilmente argomenti in caso di bisogno. Queste categorie, già citate in precedenza, definite da Cicerone e Quintiliano come magazzini di argomenti, vengono chiamate in greco tòpoi, in latino loci, in italiano luoghi. In altre parole, i luoghi sono

«Le fonti a partire [...] dalle quali l'oratore costruisce le sue argomentazioni. Si tratta di schemi argomentativi, e non di argomenti già compiutamente formulati, che vengono applicati ai casi specifici e ai quali possono venire ricondotti i singoli entimemi».

(Piazza, 2015, p. 65) 
Un esempio classico di luogo è quello che Aristotele chiama "del più e del meno": "se non si può attribuire un predicato alla cosa a cui più apparterrebbe, è evidente che non lo si può attribuire alla cosa a cui meno apparterrebbe»; da questo luogo egli ricava l'entimema «se neppure gli dei sanno tutte le cose, ancor più difficilmente le sapranno gli uomini» (Aristotele, Retorica, II, 23, 1397b). Altri esempi classici di luoghi sono quelli che rientrano nella categoria "del genere e della specie", di cui un esempio è la seguente affermazione di carattere induttivo: "se diverse specie di un genere $G$ posseggono una data proprietà $\mathrm{P}$, allora probabilmente $\mathrm{P}$ è una proprietà del genere $\mathrm{G}$ »; da questo luogo si possono ricavare numerosi entimemi; esso è utilizzato, come vedremo, nell'argomentazione matematica.

Nei secoli di storia della retorica, sono state prodotte numerose possibili classificazioni dei luoghi. Non è questa la sede per trattare le varie catalogazioni e distinzioni presenti nei diversi periodi storici, ${ }^{17}$ non solo perché l'impresa richiederebbe da sola sforzi che esulano dai fini del presente contributo, ma anche perché un elenco completo di tutti i luoghi utilizzabili in un'argomentazione è difficilmente realizzabile (Perelman \& Olbrecths-Tyteca, 1958/2013, p. 93). In questo lavoro segnaliamo il tentativo di organizzazione proposto in Rigotti e Greco (2019), che presenta il vantaggio di tentare un equilibrio tra la specificità e la precisione, ma anche l'eccessiva lunghezza, delle liste di luoghi proposte da alcuni studiosi (ad esempio Walton et al., 2008), e la sintesi e duttilità, ma anche la spesso estrema condensazione, di altre (ad esempio Whately, 1828/1963). ${ }^{18}$

\subsubsection{La dispositio: l'organizzazione del discorso}

La dispositio riguarda l'organizzazione del discorso persuasivo; essa si occupa dei vari modi con cui disporre gli argomenti in maniera che il discorso risulti efficace e persuasivo.

Nella retorica tradizionale la dispositio riguardava tre livelli:

1. la partizione dell'intero discorso e di singole sezioni;

2. I'ordinamento dei contenuti all'interno di ciascuna parte;

3. I'ordine delle parole nella formulazione delle idee.

Per quanto riguarda il primo livello, classicamente la divisione persuasiva del discorso pubblico era composta da quattro parti: esordio (l'inizio, il preambolo del discorso, nel quale può venire anche esposta la quaestio, cioè l'argomento specifico da trattare), narrazione (l'esposizione dei fatti), argomentazione (il cuore del discorso persuasivo, in cui si adducono le prove), epilogo (la conclusione del discorso). Questa suddivisione è semplificata dallo stesso Aristotele, il quale afferma che «le parti del discorso sono due: è infatti necessario esporre il fatto di cui si parla e il dimostrarlo» (Aristotele, Retorica, III, 13, 1414a). Anche Perelman e Olbrechts-Tyteca (1958/2013, p. 533) fanno notare che, in un discorso espositivo-dimostrativo, quale è ad esempio quello dei trattati di geometria, le parti del discorso si riducono solitamente alla prima e la terza (eventualmente anche con ordine invertito). Per quanto riguarda il secondo livello, Piazza fa notare che per Aristotele

«[...] l'ordine con cui si espongono gli entimemi ha [...] un peso nella persuasività complessiva del discorso e lo stesso vale, precisa Aristotele, per la quantità. Un eccesso di sillogismi non solo indebolirà l'impatto emotivo ma andrà anche a scapito sia della chiarezza sia della stessa credibilità dell'oratore».

(Piazza, 2015, pp. 161-162)

\footnotetext{
17. Per un interessante approfondimento riguardante le varie strutturazioni di luoghi avvenute nel corso della storia, rimandiamo a Rigotti e Greco (2019).

18. Le riflessioni e le proposte presenti in Rigotti e Greco (2019) fanno riferimento a un metodo di analisi dell'argomentazione - Argumentum Model of Topics (AMT) - sviluppato all'interno dell'Istituto di argomentazione, linguistica e semiotica (IALS) dell'Università della Svizzera Italiana di Lugano. Tale modello consente di portare alla luce le varie componenti che entrano in gioco in una qualsiasi inferenza argomentativa; molto affascinante è vedere come questo modello possa essere applicato anche per analizzare dimostrazioni matematiche, come mostrato in Bellucci e Blotti (2017) e Maggi (2017).
} 
Nella visione retorica classica si riteneva che, nel caso di argomentazioni multiple, non concatenate, ${ }^{19}$ fossero tre i modelli possibili di disposizione dei contenuti: ordine crescente, nel quale si incomincia dagli argomenti deboli per lasciare in ultima posizione quelli più forti, con il rischio però che gli ascoltatori siano sfavorevolmente disposti fin dall'inizio; ordine decrescente, nel quale si procede all'opposto presentando per primi gli argomenti più forti e mettendo quelli meno convincenti in secondo piano, con il rischio però che si produca nell'ascoltatore un'impressione sfavorevole a causa del fatto che le ultime cose ascoltate siano le sole a rimanere a mente; ordine omerico o nestoriano, il più raccomandato, nel quale si pongono gli argomenti più solidi all'inizio e alla fine, distribuendo nel mezzo le ragioni meno forti. Al di fuori di questi tre modelli, che riguardano la disposizione degli entimemi in un discorso persuasivo, Aristotele tratta gli esempi, affermando che occorra

«[...] qualora se ne posseggano, servirsi di essi come testimonianze, usandoli come epilogo per gli entimemi: infatti se sono anteposti danno l'impressione di un'induzione, e per i discorsi retorici I'induzione non è appropriata se non in pochi casi, se sono detti a conclusione invece fungono da testimonianze, e la testimonianza è in ogni caso persuasiva».

(Aristotele, Retorica, II, 20, 1394a)

Nella sistemazione in "casi particolari" di Perelman e Olbrechts-Tyteca (1958/2013), questo condurrebbe ad affermare che, nei discorsi persuasivi, l'illustrazione è più efficace dell'esempio; in realtà, se si considera che l'approccio moderno alle varie tecniche argomentative si rifiuta di considerare l'argomentazione come un discorso che trova in sé stesso la sua struttura ideale (l'efficacia infatti dipende dal particolare uditorio a cui ci si rivolge) allora occorre concludere che "devono essere le esigenze dell'adattamento all'uditorio a guidare nello studio dell'ordine del discorso» (Perelman \& Olbrechts-Tyteca, 1958/2013, p. 547).

Quanto all'ultimo punto, infine, quello relativo cioè alla disposizione delle parole nella formulazione delle idee, esso presenta forti intrecci con la fase dell'elocutio, ma può essere interessante ricordare brevemente che esistono forme "non marcate" e forme "marcate" di disporre le parole. In breve, una forma "non marcata" rappresenta uno standard linguistico, un uso comune della disposizione delle parole che non presenta elementi che catturano particolarmente l'attenzione di chi legge o ascolta, risultando per certi versi neutrale e appropriato alla maggior parte dei contesti. Una forma "marcata" è al contrario caratterizzata da una scelta non di default, che presuppone un'intenzione di enfatizzazione da parte di chi la usa, e che risulta meno scontata e non neutrale. Ad esempio, in Rocci (1996) si fa notare che nel sintagma nominale italiano gli aggettivi possono tanto precedere quanto seguire il nome, ma l'ordine "non marcato" prevede tipicamente che l'aggettivo venga dopo il nome (la frase "la penna rossa!" è generalmente più neutrale e "non marcata" di "la rossa penna!").

\subsubsection{L'elocutio: scelta della forma linguistica del discorso}

L'elocutio riguarda il modo, lo stile con il quale viene presentato il discorso argomentativo: si occupa di dare forma linguistica alle idee in modo che risultino il più efficaci possibile.

Tradizionalmente, il materiale linguistico oggetto di elaborazione nell'elocutio è suddiviso in parole singole e connessioni di parole; per ciascuno di questi due gruppi vengono esercitate le quattro qualità (virtus in latino) dello stile oratorio: I'aptum, ovvero l'appropriatezza, che corrisponde al requisito di un discorso di essere conforme, che si convenga cioè alle specifiche circostanze e agli scopi nel quale viene prodotto; la puritas, ovvero la correttezza lessicale e grammaticale, che si fonda sul rispetto di un'ideale di integrità della lingua; la perpiscuitas, ovvero la chiarezza, la nitidezza del discorso, neces-

19. Le argomentazioni multiple sono argomentazioni che prevedono più argomenti coordinati e direttamente operativi a sostegno di una stessa opinione. 
saria affinché esso sia comprensibile; infine l'ornatus, ovvero l'eleganza, la bellezza derivante dall'uso opportunamente regolato di mezzi e ornamenti linguistici rappresentati dalle figure retoriche.

Per i nostri scopi è utile approfondire, seppur in modo non esaustivo, il tema della chiarezza espositiva nell'elocutio, cioè la perspicuitas latina. In primo luogo occorre osservare che l'essere chiaro e comprensibile è una qualità soggetta alla valutazione del particolare uditorio cui il discorso si riferisce. In effetti, come osserva Mortara Garavelli

«[...] diversamente dalla puritas, per cui esisteva una base grammaticale obiettiva per riconoscere ciò che era vietato e ciò che era permesso, la perspicuitas e i suoi contrari venivano definiti, dalla precettistica dell'arte oratoria, in base al criterio principe dell'adattamento all'uditorio».

(Mortara Garavelli, 1988/2020, p. 192)

Il massimo difetto di chiarezza si ha quando il discorso risulta totalmente oscuro e incomprensibile, ad esempio perché composto in una lingua ignota all'uditorio. Se ci mettiamo nell'ottica dell'apprendimento della matematica, apprendimento che è costituito anche dall'imparare termini ed espressioni del suo linguaggio specialistico (Lavinio, 2004), può ad esempio accadere, soprattutto nei livelli iniziali di scolarità, che l'alunno senta un discorso o un testo come oscuro a causa della non conoscenza di alcune delle parole che vengono usate. Al di là di questo estremo, le oscurità parziali del discorso possono riguardare la presenza di passaggi impliciti, oppure le due categorie dell'ambiguità semantica e dell'ambiguità sintattica. La prima riguarda la presenza di termini o di costrutti grammaticali che possono essere interpretati in modi diversi, qual è il caso delle parole polisemiche ${ }^{20}$ come "angolo", usato in matematica per indicare un ente geometrico, nella lingua comune per indicare, ad esempio, il punto di incontro di due o più spigoli di una parete. La seconda riguarda quelle frasi passibili di più interpretazioni e nasce dalle relazioni tra le parole e le componenti sintattiche di una frase; ad esempio, la frase «I poligoni regolari hanno tutti i lati e tutti gli angoli uguali», presenta un'ambiguità sintattica, perché la parola "uguali" posta alla fine potrebbe essere intesa nel senso di "lati e angoli uguali fra loro", cosa che tra l'altro potrebbe generare confusione nella mente di chi legge, considerando che non ha senso mettere in relazione di uguaglianza due entità diverse quali sono i lati e gli angoli di un poligono.

Quanto all'eccesso opposto, ovvero alla ricerca esagerata di chiarezza, essa è variabile e sostanzialmente dipende dai tipi di discorso: un buon esempio è quando il discorso risulta pedante a causa di precisazioni superflue, perché l'uditorio è già esperto del tema di cui si sta trattando.

\subsection{Breve digressione multimodale}

Un ultimo punto da dover trattare prima di addentrarci nell'analisi, vista la natura dei testi di cui ci occuperemo, riguarda il fatto che le sopra esposte categorie dello studio di un discorso possano essere applicate nel caso di testi multimodali, nei quali cioè sono presenti differenti modi semiotici, non solo di tipo linguistico: immagini, scritture, simboli, elementi grafici di varia natura. Oggigiorno, infatti, il testo è raramente presentato nella sua purezza; anzi, tipicamente è solo parte di un dispositivo che contiene al suo interno altri elementi formattati insieme, in un tutto organico. Anche il libro di testo scolastico è oggi scritto e presentato con la dovuta formattazione grafica; questo accade a maggior ragione con la matematica, e soprattutto con la geometria, che prevede inevitabilmente al suo interno, accanto a quella linguistica, anche una dimensione figurale e grafica caratteristica. ${ }^{21}$ La prospetti-

20. Uno studio di questi aspetti è presentato in Demartini, Fornara e Sbaragli (2020), in cui si sono messe in luce le problematiche didattiche relative alla presenza di parole polisemiche nell'apprendimento di concetti matematici.

21. Ė riconosciuto dalla ricerca in didattica che il linguaggio della matematica sia un sistema semiotico multimodale che include testi verbali, espressioni simboliche e rappresentazioni figurali, e multivariato, che include un ampio spettro di registri (P. L. Ferrari, 2021). 
va di analizzare le argomentazioni prodotte in ambito multimodale è relativamente recente (si veda ad esempio Kjeldsen, 2015; Pollaroli \& Rocci, 2015; Rocci, 2017; Rocci \& Pollaroli, 2018) e sfrutta strumenti provenienti tanto dalla tradizione degli studi linguistici e di argomentazione, quanto le attuali scienze della comunicazione relative all'uso delle immagini e degli espedienti grafici. Come vedremo meglio nei prossimi paragrafi, per i nostri scopi è sufficiente riconoscere che, all'interno di discorsi argomentativi, I'uso di elementi e strategie semiotiche non linguistiche, quali sono ad esempio le figure geometriche, I'uso di frecce o del colore, l'organizzazione del layout di una pagina, può assolvere a diverse funzioni che rientrano nelle materie dell'inventio, dispositio ed elocutio. ${ }^{22} \mathrm{Ad}$ esempio, la scelta di rappresentare uno specifico poligono invece che un altro, oppure di rappresentarne più di uno, può essere interpretata in termini di scelta degli argomenti che sostengono in modo più o meno efficace la tesi conclusiva all'interno dell'argomentazione, rientrando dunque nell'inventio del discorso; la scelta di disporre le figure e il testo all'interno della pagina in un modo piuttosto che in un altro può dar luogo a risultati comunicativi più o meno efficaci, rientrando dunque nella dispositio del discorso; infine la scelta stessa di rappresentare una data procedura o fatto geometrico secondo due modi semiotici diversi, quello linguistico e quello figurale, può essere intesa relativa al modo e alla forma con le quali presentare il discorso per far sì che esso risulti più comprensibile per I'uditorio, rientrando dunque nel tipo di scelte interne all'elocutio.

\section{Analisi delle scelte di inventio, dispositio ed elocutio riscontrate nel caso di studio}

In questo paragrafo riprendiamo il caso di studio presentato nel par. 2, relativo a un libro di testo italiano del primo anno di scuola secondaria di primo grado, e lo interpretiamo con le chiavi di lettura dell'inventio, della dispositio e dell'elocutio. Per comodità ne riportiamo nuovamente l'immagine.

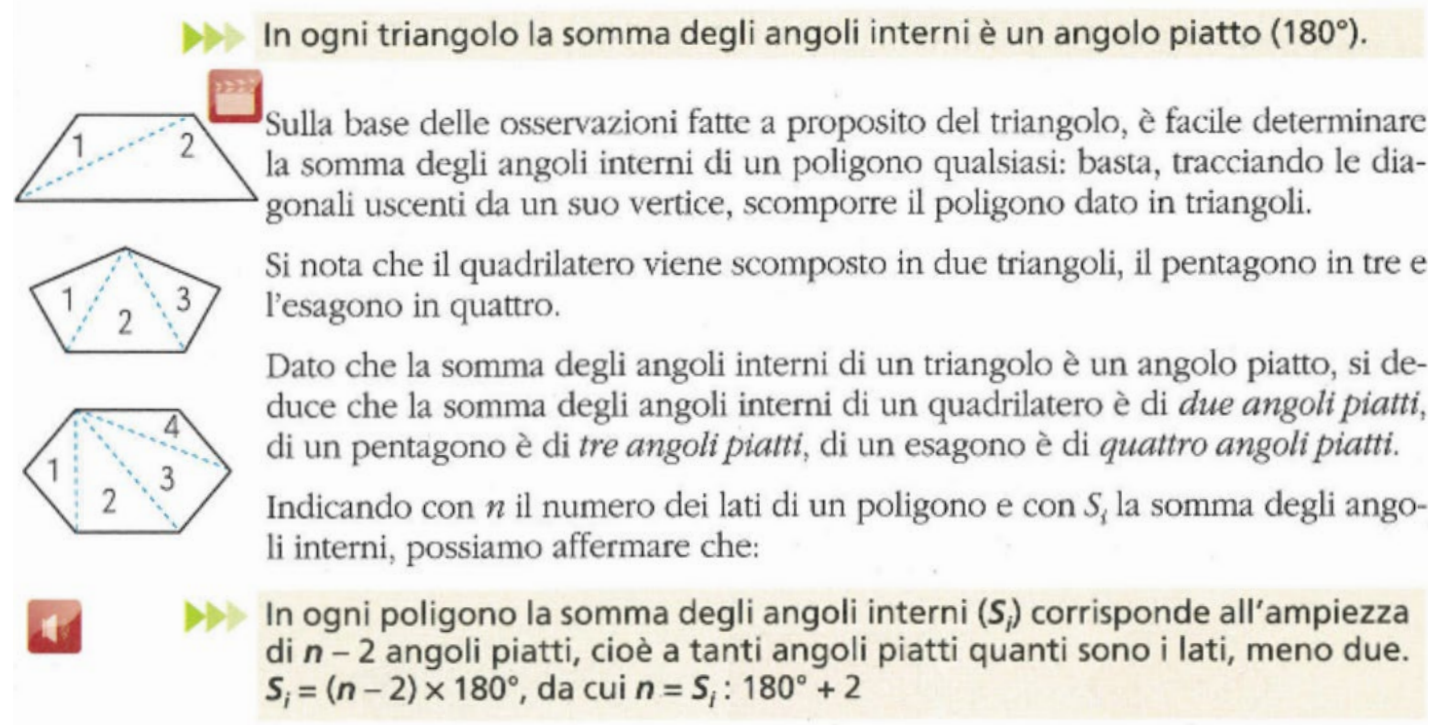

22. Non solo: in Canducci, Rocci e Sbaragli (2021), si mostra come I'utilizzo più o meno efficace delle strategie semiotiche multimodali nei libri di testo di geometria possa portare a problematiche significative per il lettore-allievo, ad esempio quando tali strategie sono utilizzate per favorire il processo di conversione semiotica tra rappresentazioni espresse in registri diversi (Duval, 1993). 


\subsection{L'inventio nel caso di studio}

Un primo livello di inventio riscontrato in questa porzione di testo riguarda la tipologia di costruzione geometrica scelta come base della strategia argomentativa, che si basa sulla triangolazione del poligono effettuata tracciando tutte le diagonali che hanno come estremo un unico vertice fissato (inventio 11). Attraverso questa costruzione, la strategia argomentativa consiste nel mostrare che un poligono di $n$ lati viene scomposto in $n-2$ triangoli, per poi inferire che la somma delle ampiezze degli angoli interni di un poligono è data dalla moltiplicazione tra $n-2$ e $180^{\circ}$, giungendo così alla tesi conclusiva.

A livello di tipo di ragionamento proposto, I'estratto rientra nella tipologia di inventio induttiva basata sull'esempio individuato da Aristotele: per dare fondamento al fatto che, triangolando in questo modo, un poligono di $n$ lati viene suddiviso in $n-2$ triangoli, vengono forniti esempi in cui ciò accade. In primo luogo notiamo, a livello quantitativo, che il testo sceglie di fornire tre esempi: questa scelta è importante perché, banalmente, può essere vista come relativa alla quantità di premesse su cui basare l'argomentazione: ci si potrebbe chiedere se l'argomentazione sarebbe stata più o meno efficace se fosse stato presentato su un numero maggiore o minore di esempi. Anche la scelta di quali esempi fornire rientra nella materia dell'inventio: in questo caso si è scelto di riferirsi a un quadrilatero, un pentagono e un esagono, ossia ai tre poligoni che seguono il triangolo nella successione dei tipi di poligoni in base ai lati, scelta basata sulla speranza di favorire una generalizzazione che non sarebbe stata facilitata scegliendo, ad esempio, un ettagono, un dodecagono e un icosagono. La successione dei tipi dei poligoni sembra infatti ricalcare un processo di ricerca di una regola, di uno schema, che valga in generale e che prevede, almeno inizialmente, di esplorare casi in qualche modo vicini tra loro, con il fine di rinforzare una convinzione che si sta costruendo in modo empirico.

Parlando di generalizzazione, sempre a livello di inventio, notiamo che sono presenti almeno tre luoghi principali. Un primo luogo, utilizzato qui in senso induttivo, rientra nella categoria "del genere e della specie", già citato nel par. 3.1.1: "se diverse specie di un genere G posseggono una data proprietà $\mathrm{P}$, allora probabilmente $\mathrm{P}$ è una proprietà del genere $\mathrm{G}$ "; questo luogo viene utilizzato per generalizzare a un poligono generico tre proprietà: la proprietà relativa al tipo di triangolazione, quella relativa al numero di triangoli che si ottengono con tale triangolazione e quella relativa al fatto che il numero di triangoli così ottenuti sia pari al numero dei lati del poligono meno due.

Un secondo luogo rientra all'interno della categoria "della specie e dell'individuo", ed è invece utilizzato in modo deduttivo: "Se per la specie S vale una proprietà P, allora per ogni individuo della specie $S$ vale la proprietà $\mathrm{P}$ ", ed è utilizzato quando si applica il teorema relativo alla somma degli angoli interni di un triangolo generico ai triangoli ottenuti dalla costruzione. Un terzo luogo rientra invece nella categoria "parte e tutto": "se un tutto è composto dal numero $X$ di parti $P$ uguali rispetto alla grandezza $\mathrm{Q}$, allora il valore di $\mathrm{Q}$ per il tutto corrisponderà a $\mathrm{X}$ volte $\mathrm{P}$ ", ed è utilizzato per calcolare la somma delle ampiezze degli angoli interni di un poligono come $n$ - 2 volte la somma delle ampiezze degli angoli interni di un triangolo. Osserviamo in generale che l'eterogeneità e la quantità di elementi di inventio qui richiamati rendono conto di una complessità che ci sembra non evidente a una lettura limitata, che non prevede l'impostazione qui adottata: lo sguardo proposto si aggiunge dunque a quello abituale in didattica della matematica, arricchendolo.

\subsection{La dispositio e l'elocutio nel caso di studio}

Guardiamo ora a come è composto l'estratto a livello di dispositio, inserendo all'interno del commento anche riflessioni riguardo allo stile e alla forma linguistica con la quale essa viene realizzata - cioè all'elocutio. In prima battuta facciamo notare che, dal punto di vista matematico, la tesi è posta in fondo alla pagina e che, dal punto di vista dell'organizzazione del discorso trattata nel par. 3.1.2, I'estratto può essere suddiviso nelle tre parti: esordio, argomentazione, epilogo. Notiamo che non è presente la parte della narrazione, che tipicamente si riferisce a discorsi in cui è necessario esporre 
eventi e avvenimenti riguardanti personaggi ed episodi significativi ai fini dello sviluppo dell'argomentazione.

Esordio. L'esordio è composto dal primo enunciato del primo blocco testuale: vengono richiamate premesse che verranno utilizzate nell'argomentazione (le "osservazioni fatte a proposito del triangolo"); viene inoltre manifestata la quaestio, cioè il tema su cui verterà l'argomentazione ("determinare la somma degli angoli interni di un poligono qualsiasi") spingendosi a dichiarare la semplicità della sua risoluzione ("è facile") con l'intento, forse, di disporre benevolmente il lettore.

Argomentazione. II ragionamento argomentativo inizia nel primo blocco subito dopo la manifestazione della quaestio, alla quale si collega attraverso il segno di interpunzione forte ":". I due punti hanno qui una funzione esplicativa, e sono eloquentemente seguiti dal predicato verbale "basta", il cui significato da un lato rafforza in chi legge l'impressione relativa alla facilità di quanto si andrà a trattare, dall'altro ha la funzione di introdurre la costruzione alla base della strategia di inventio 11 ("tracciando le diagonali uscenti da un suo vertice, scomporre il poligono dato in triangoli").

Il testo prosegue, nel secondo blocco testuale, esemplificando la costruzione appena enunciata: il ragionamento, proposto sulla base di tre esempi riguardanti "il quadrilatero", "il pentagono" e "I'esagono", è introdotto dal sintagma "si nota che", e viene accompagnato da tre rappresentazioni figurali poste a sinistra. L'enunciato afferma che "il quadrilatero viene scomposto in due triangoli, il pentagono in tre e l'esagono in quattro", affermazione sostenuta, a livello di elocutio multimodale, anche dalla scelta di rappresentare all'interno delle figure la notazione indo-araba del numero dei triangoli che si ottiene in ciascuno dei tre casi. È interessante richiamare che le scelte di dispositio operate in questo blocco seguono un ordine sequenziale rispetto al numero di lati. Questo è significativo perché, a differenza dell'induzione matematica in cui occorre mostrare la relazione tra il caso $n$-esimo e il caso $(n+1)$-esimo, il procedimento argomentativo di tipo induttivo in sé non necessita di una gradualità nell'ordine degli esempi che vengono proposti; questa scelta appare dunque tanto di tipo retorico quanto didattico-matematica: chi ha scritto questo estratto ha giustamente ritenuto più convincente presentare gli esempi in ordine crescente rispetto al numero dei lati, facendo corrispondere all'induzione argomentativa quella matematica. Dal punto di vista figurale, facciamo inoltre notare che il quadrilatero, il pentagono e l'esagono non sono generici: il primo è un trapezio isoscele; gli altri due, pur non essendo regolari, presentano simmetrie assiali. Se si considera che nella parte linguistica si parla genericamente di quadrilatero, pentagono ed esagono (introdotti dall'articolo con valore generico "il"), questa scelta di elocutio multimodale potrebbe essere da un certo punto di vista interpretata come scelta di tipo estetico, legata cioè all'ornatus, ma dal punto di vista pedagogico appare discutibile, perché il lettore potrebbe implicitamente assumere che la triangolazione proposta sia valida solo quando i poligoni presentano particolari caratteristiche geometriche. Sempre in questo blocco testuale, infine, non si può trascurare il fatto che, a livello di elocutio, esso presenti elementi di oscurità parziale dati dalla scelta di mantenere impliciti sia la relazione fra numero di lati e numero di triangoli che si ottengono dalla triangolazione per i tre esempi (4 lati - 2 triangoli; 5 lati - 3 triangoli; 6 lati - 4 triangoli), sia la generalizzazione al poligono generico dell'uguaglianza: "numero di triangoli = numero dei lati meno due". Questa scelta di elocutio appare ovviamente problematica non solo ai fini di una chiarezza espositiva ma anche ai fini di un accompagnamento del lettore alla comprensione dell'argomentazione proposta. Ipotizziamo che tale scelta di de-enfatizzazione nasconda una certa ritrosia, da parte del libro di testo, nell'esplicitare che quanto si sta proponendo non sia una vera e propria dimostrazione rigorosa, ma presenta un carattere induttivo che, forse, agli occhi del lettore, potrebbe risultare meno convincente. La scelta di lasciare impliciti i passi induttivi sembra in qualche modo mascherare la strategia argomentativa adottata, ma allo stesso tempo potrebbe condurre il lettore-allievo a considerarla come dimostrazione vera e propria. Se così fosse, l'allievo potrebbe perdere un'occasione per rendere cosciente a sé stesso l'esistenza di prove di natura diversa da quel- 
le deduttive, formalmente non accettate in matematica, ma molto utili nella dimensione didattica dell'apprendimento della disciplina. ${ }^{23} \mathrm{D}^{\prime}$ altra parte, se è vero che in un'argomentazione è opportuno dire né troppo né troppo poco, ci troviamo davanti a un dilemma tipico della perspicuitas retorica: quanto esplicitare, quanto dire, perché il discorso risulti chiaro e allo stesso tempo non pedante o eccessivamente complesso per l'uditorio?

II terzo blocco testuale presenta l'argomento, di carattere deduttivo, con il quale si applica il teorema della somma degli angoli interni di un triangolo per dedurre la somma degli angoli interni di un quadrilatero, di un pentagono e di un esagono. Richiamiamo l'attenzione sul fatto che in questo blocco, e solo in questo, viene utilizzato il corsivo nei sintagmi "due angoli piatti", "tre angoli piatti", "quattro angoli piatti", cioè il numero di angoli piatti cui corrisponde la somma degli angoli interni del quadrilatero, del pentagono e dell'esagono. Questa scelta di enfatizzazione rientra tipicamente nel tipo di scelte di elocutio che possono essere effettuate in un testo scritto, anche se in questo caso la sua efficacia può essere messa in dubbio: perché richiamare l'attenzione visiva solo sui risultati, e non sulla relazione tra poligono specifico e numero di angoli piatti, ad esempio utilizzando un colore per la coppia quadrilatero-due angoli piatti, un altro per la coppia pentagono-tre angoli piatti, un altro ancora per la coppia esagono-quattro angoli piatti?

Il quarto e penultimo blocco testuale rientra ancora nella parte argomentativa del discorso; interessante è notare che viene esplicitato per la prima volta il simbolismo matematico $\left(n\right.$ e $\left.S_{i}\right)$ che consentirà di esprimere la tesi in termini di un'uguaglianza simbolica; questa scelta di dispositio non è l'unica possibile, perché si potrebbe scegliere, ad esempio, di introdurre il simbolismo necessario all'inizio dell'argomentazione: da un lato la scelta di introdurlo alla fine sembra sorreggere una maggiore fluidità nella discorsività del ragionamento; dall'altro, il rischio è di appesantire in modo repentino e non graduale l'argomentazione, aumentando la complessità semantica per il lettore, il quale deve saper improvvisamente decodificare una forma linguistico-simbolica nuova, che non è rientrata fino a questo momento nello stile di elocutio globale del discorso.

Epilogo. Infine, I'ultimo blocco testuale presenta la conclusione del discorso, nella quale viene esposta la tesi cui si voleva giungere. Osserviamo in primo luogo come questa conclusione venga inserita all'interno di un box colorato, segnalato a sua volta da tre triangoli di colore verde posti di fianco; queste scelte rientrano nella categoria di elocutio, perché hanno l'effetto di richiamare l'attenzione visiva del lettore attraverso delle enfasi di tipo semiotico multimodale date dall'uso di un colore diverso dallo sfondo bianco del testo precedente e dall'uso di elementi deittici extra-linguistici. Se ci concentriamo poi sulla parte testuale, si osserva la scelta di ribadire la tesi per ben tre volte con formulazioni diverse. Due di queste sono interne al primo enunciato, suddiviso in due proposizioni: la prima ("In ogni poligono la somma degli angoli interni $\left(S_{i}\right)$ corrisponde all'ampiezza di $n-2$ angoli piatti") è una formulazione in parte linguistica e in parte simbolica, nella quale vengono richiamati i simboli introdotti nel blocco precedente; la seconda ("a tanti angoli piatti quanti sono i lati, meno due") è una formulazione esclusivamente linguistica che segue subito dopo la congiunzione con valore riformulativo "cioè". A questo enunciato segue, dopo la pausa data dal punto, una terza formulazione (" $S_{i}=(n-2) \cdot 180^{\circ}$ ") esclusivamente simbolica. ${ }^{24}$ Non possiamo fare a meno di mettere in evidenza l'abbondanza delle rappresentazioni, che da un lato è positiva perché stimola processi cognitivi imprescindibili nell'apprendimento della matematica (il trattamento e la conversione semi-

23. Questa de-enfatizzazione potrebbe anche essere interpretata come scelta efficace da parte del libro di testo: sostare in modo esplicito sull'utilizzo di un procedimento induttivo potrebbe condurre lo studente a pensare che si tratti di un contenuto da apprendere, e non di una strategia pedagogica per la costruzione di congetture matematiche.

24. L'ulteriore espressione simbolica nel testo $\left(n=S_{i}: 180^{\circ}+2\right)$, introdotta dal connettivo "da cui", rappresenta una tipica, e spesso malsana, abitudine, molto presente nei libri di questo livello scolastico, di presentare immediatamente le "formule inverse" dei risultati appena esposti senza ulteriori spiegazioni. Pur essendo questa scelta potenzialmente veicolo di confusione da parte del lettore, non commenteremo questo ulteriore passaggio, limitandoci a osservare come, ponendolo alla fine dell'argomentazione, gli si stia dando un'importanza forse non desiderata. 
otica secondo la teoria di Duval, 1993); dall'altro, in questo caso, ci domandiamo in primo luogo se non vi sia il rischio che queste tre enunciazioni possano essere intese come enunciati semanticamente diversi tra loro, e in secondo luogo se queste diverse rappresentazioni potessero essere disposte in modo più efficace, ad esempio separando in modo netto le prime due, oppure presentando prima la formulazione esclusivamente linguistica, poi quella mista, infine quella simbolica. In ultimo osserviamo che la formulazione linguistica "cioè a tanti angoli piatti quanti sono i lati, meno due" conduce a un'ambiguità sintattica a causa della virgola posta tra la parola "lati" e "meno": uno studente che leggesse solo questa parte potrebbe interpretare la pausa indicata dalla virgola in senso procedurale, rischiando di capire che per trovare la somma degli angoli interni di un poligono occorra prima considerare tanti angoli piatti quanti sono i lati, e poi sottrarre due a questo valore.

Le scelte di inventio, dispositio ed elocutio apportate in questo caso di studio rispecchiano solo alcune delle variegate possibilità retoriche presenti nei libri di testo di geometria; d'altronde, questo è naturale se si considera che, in generale, la retorica può essere considerata come arte di scegliere forme e strutture del discorso in modo che esso sia funzionale agli obiettivi di ragionevolezza e persuasione che ci si pone. Nei prossimi paragrafi metteremo a confronto le scelte del caso di studio con altre presenti nel corpus di libri DFA-Italmatica mostrandone dunque la molteplicità e le differenze; ciò verrà fatto accompagnandole con alcuni dati quantitativi.

\section{La varietà di scelte nell'inventio, dispositio ed elocutio nei libri di testo}

In questo paragrafo metteremo a confronto le scelte fatte a livello di categorie retoriche di inventio e di dispositio ed elocutio nel caso di studio da noi scelto con quelle effettuate in altri libri di testo scolastici rientranti nel corpus DFA-Italmatica. Questo al fine di cogliere la grande varietà di scelte che si possono intraprendere da questo punto di vista sullo stesso tema, e il possibile impatto comunicativo che potrebbero avere sui lettori-allievi. Va considerato che dei 143 libri del corpus, sono 23 quelli nei quali viene presentato l'argomento da noi scelto: 1 italiano di quinta primaria, 20 italiani di prima secondaria di primo grado; 1 italiano di seconda secondaria di primo grado; 1 svizzero (Canton Ticino) del secondo anno di scuola media. ${ }^{25}$

\subsection{La varietà dell'inventio per la somma delle ampiezze degli angoli interni di un poligono}

Nel corpus si possono identificare tre tipi di scelte di inventio, chiaramente distinte a livello di scelta della costruzione geometrica come base della strategia argomentativa. La prima (I1) è stata presentata nel paragrafo precedente; la seconda (I2) e la terza (I3) vengono invece analizzate in questo paragrafo.

- L'inventio 12 si basa sulla triangolazione del poligono che si ottiene tracciando i segmenti che hanno come estremi un punto interno al poligono e i vertici del poligono. Tale scelta è presente nel seguente esempio (Figura 2). 


\section{$7 \quad$ Angoli dei poligoni}

La somma degli angoli interni di un poligono è uguale a tanti angoli piatti quanti sono i lati meno due.

Disegna un poligono, ad esempio un esagono, e segna un punto al suo interno.

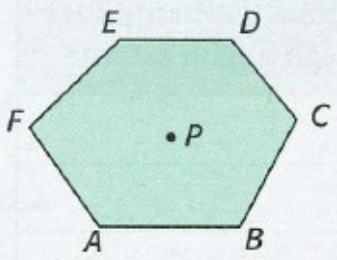

Congiungi il punto con i vertici del poligono: ottieni 6 triangoli.

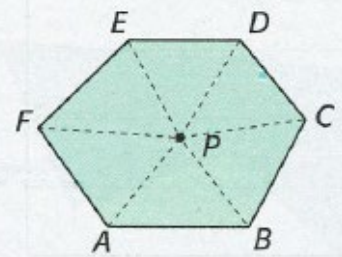

In ogni triangolo la somma degli angoli interni è un angolo piatto. La somma di tutti gli angoli segnati è 6 angoli piatti. Per avere la somma degli angoli interni del poligono devi togliere l'angolo giro (cioè due angoli piatti) con vertice in $P$.
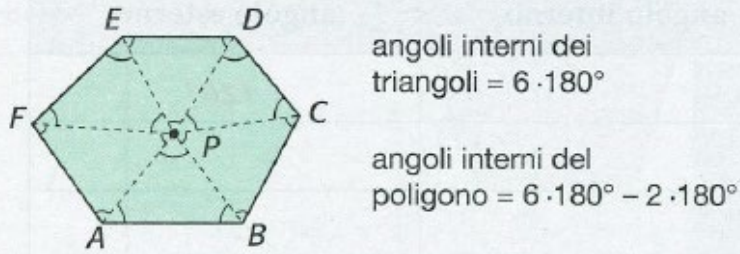

Figura 2. Esempio tratto da un libro di testo italiano del primo anno di scuola secondaria di primo grado in cui si utilizza la strategia di inventio 12 (libro 19_6 del corpus, p. 155)

Analizzando l'esempio più nello specifico, anche l'argomentazione qui proposta rientra nella tipologia di inventio del "caso particolare" individuato da Perelman e Olbrechts-Tyteca (1958/2013). Pur presentando una struttura che potrebbe far pensare alla porzione testuale in termini di illustrazione, si tratta in realtà ancora di un esempio utilizzato induttivamente per dare delle ragioni che sostengono l'accettazione della tesi, posta all'inizio del discorso. Una caratteristica a livello di inventio che emerge immediatamente è la scelta di presentare un solo esempio a sostegno dell'argomentazione, nello specifico un esagono, a differenza del caso di studio dove se ne proponevano tre diversi tra loro che conducono alla generalizzazione. La scelta di fornire un solo esempio, unita alla mancanza di passi linguistici che potrebbero esplicitare le relazioni tra il caso specifico dell'esagono e il caso generico del poligono di $n$ lati, sembra rendere ancora più nascosta rispetto al caso di studio la generalizzazione dal caso particolare al caso di un poligono generico, generalizzazione che sembra essere totalmente a carico del lettore. A livello di luoghi, ne notiamo almeno tre, afferenti alle categorie "della specie e dell'individuo" e "parte e tutto". II primo, usato in modo analogo al caso di studio, è di carattere deduttivo e rientra nella categoria "della specie e dell'individuo" ("Se per la specie $\mathrm{S}$ vale una proprietà $\mathrm{P}$, allora per ogni individuo della specie $\mathrm{S}$ vale la proprietà $\mathrm{P}$ "). Un secondo luogo, anch'esso utilizzato nel caso di studio, e che rientra nella categoria "parte e tutto" è usato per cal- 
colare la somma delle ampiezze degli angoli interni dei triangoli ottenuti dalla triangolazione come $n$ volte la somma delle ampiezze degli angoli interni di un triangolo; sempre in questa categoria può rientrare anche un terzo luogo: "se un tutto T è composto dalle parti $A$ e $B$, allora una delle due parti corrisponde al tutto meno l'altra parte", utilizzato per individuare la somma delle ampiezze degli angoli interni del poligono come differenza fra la somma delle ampiezze degli angoli di tutti i triangoli ottenuti dalla triangolazione e l'ampiezza dell'angolo giro.

- L'inventio I3 si basa invece su una costruzione incentrata sulla constatazione che gli angoli interni ed esterni sono supplementari. Tale costruzione si riscontra nel seguente esempio (Figura 3).

\section{Relazioni tra gli angoli interni}

Osserva il poligono e i suoi angoli interni. Si nota che ogni angolo interno con il suo corrispondente esterno forma un angolo piatto:

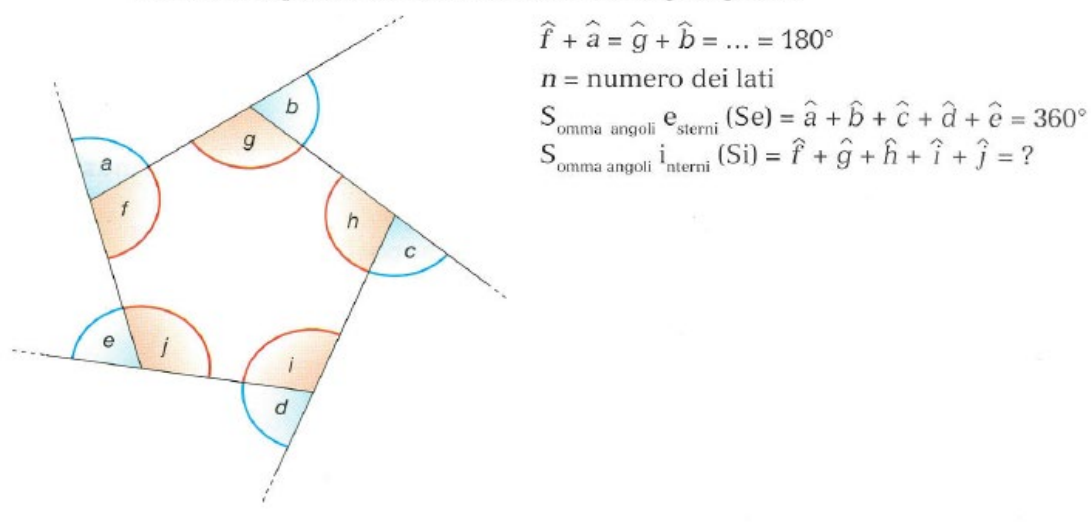

Quindi, in questo poligono che ha cinque lati, la somma degli angoli interni (Si) e dei suoi angoli esterni (Se) è uguale a 5 angoli piatti:

$$
\mathrm{Si}+\mathrm{Se}=5 \times 180^{\circ}
$$

Poiché la somma degli angoli esterni è uguale a un angolo giro, cioè a due angoli piatti, per calcolare la somma degli angoli interni del poligono basta togliere 2 angoli piatti dalla somma di tutti gli angoli ( $\mathrm{Si}+\mathrm{Se})$, cioè:

$$
\mathrm{Si}=5 \times 180^{\circ}-2 \times 180^{\circ}=(5-2) \times 180^{\circ}=3 \times 180^{\circ}
$$

La somma degli angoli interni di un pentagono è uguale a 3 angoli piatti, cioè $540^{\circ}$. Questo ragionamento può essere fatto per qualsiasi poligono; si ha che:

la somma delle ampiezze degli angoli interni di un poligono qualsiasi
è uguale a tanti angoli piatti quanti sono i lati meno due.

Indicando con $n$ il numero dei lati, si può scrivere la formula per calcolare la somma degli angoli interni:

$$
\mathrm{Si}=(n-2) \times 180^{\circ}
$$

Figura 3. Esempio, tratto anch'esso da un libro di testo italiano del primo anno di scuola secondaria di primo grado, in cui si utilizza la strategia di inventio 13 (libro C1_6, p. 193).

A livello di tipo di ragionamento proposto, anche questo estratto rientra nella tipologia di inventio del "caso particolare", nello specifico in quello che gli autori chiamano esempio, anche se a differenza di quanto accadeva per il caso di studio, si sceglie nuovamente, in analogia a quanto scelto nell'esempio relativo all'inventio 12 , di fornire un solo esempio a sostegno dell'argomentazione, sviluppato attorno alla costruzione relativa a un pentagono. Per quanto riguarda i luoghi presenti nel testo, ne troviamo almeno quattro, afferenti a tre categorie presenti anche nel caso di studio e nell'esempio precedente. Il primo, interno alla categoria "della specie e dell'individuo" ("Se per la specie S vale una proprietà $\mathrm{P}$, allora per ogni individuo della specie S vale la proprietà $\mathrm{P}$ ") è utilizzato quando si applica al caso del pentagono il fatto che la somma delle ampiezze degli angoli esterni di un poligono corrisponde 
all'ampiezza di un angolo giro. Un secondo luogo, anch'esso utilizzato nell'esempio precedente, è qui usato per calcolare la somma delle ampiezze degli angoli interni ed esterni del pentagono come $n$ volte la somma delle ampiezze di un angolo interno ed esterno corrispondente (ossia 180'), e rientra nella categoria "parte e tutto"; sempre in questa categoria rientra anche il terzo luogo, presente anche nell'esempio precedente: "se un tutto T è composto dalle parti A e B, allora una delle due parti corrisponde al tutto meno l'altra parte", qui utilizzato per individuare la somma delle ampiezze degli angoli interni del pentagono come differenza fra la somma delle ampiezze degli angoli di tutti i triangoli ottenuti dalla triangolazione e l'ampiezza dell'angolo giro. Infine, troviamo in questo estratto anche un luogo "del genere e della specie", già citato nel caso di studio: "se diverse specie di un genere $G$ posseggono una data proprietà $P$, allora probabilmente $P$ è una proprietà del genere $G$ "; questo luogo viene utilizzato per generalizzare a un poligono generico il ragionamento adottato in questo caso, come manifestato dall'affermazione presente nel testo "Questo ragionamento può essere fatto per qualsiasi poligono". Questa affermazione risulta cruciale, in quanto rende esplicito il passo di generalizzazione che non è coperto dalle altre inferenze di tipo deduttivo presenti nel discorso.

Gli esempi mostrati relativi alle tre strategie di inventio presentano dunque elementi di diversità al loro interno, dati dalla scelta del tipo di ragionamento proposto e da diverse ulteriori scelte. Nella Tabella 1 vengono schematizzate le differenze più evidenti che fanno riferimento alle conoscenze matematiche in gioco per ciascuna delle tre strategie. Abbiamo categorizzato queste differenze dal punto di vista dei prerequisiti geometrici necessari, dei risultati matematici nuovi cui si giunge e dei passaggi aritmetico-algebrici coinvolti.

\begin{tabular}{|c|c|c|c|}
\hline & Inventio I1 & Inventio 12 & Inventio 13 \\
\hline \multirow[t]{3}{*}{ Prerequisiti } & \multirow[t]{3}{*}{$\begin{array}{l}\text { La somma delle ampiezze } \\
\text { degli angoli interni di un } \\
\text { triangolo è } 180^{\circ} \text {. }\end{array}$} & $\begin{array}{l}\text { La somma delle ampiezze } \\
\text { degli angoli interni di un } \\
\text { triangolo è } 180^{\circ} \text {. }\end{array}$ & $\begin{array}{l}\text { La somma delle ampiezze } \\
\text { degli angoli esterni di un } \\
\text { poligono è costante ed è } \\
360^{\circ} \text {. }\end{array}$ \\
\hline & & $\begin{array}{l}\text { Un angolo giro ha } \\
\text { un'ampiezza di } 360^{\circ} \text {. }\end{array}$ & \multirow[t]{2}{*}{$\begin{array}{l}\text { Un angolo giro corrisponde } \\
\text { a due angoli piatti. }\end{array}$} \\
\hline & & $\begin{array}{l}\text { Un angolo giro corrisponde } \\
\text { a due angoli piatti. }\end{array}$ & \\
\hline \multirow[t]{3}{*}{ Risultati matematici } & $\begin{array}{l}\text { Il numero di triangoli } \\
\text { ottenuti dalla triangolazione } \\
\text { è pari al numero dei lati del } \\
\text { poligono }(n) \text { meno due. }\end{array}$ & $\begin{array}{l}\text { Il numero dei triangoli } \\
\text { ottenuti dalla triangolazione } \\
\text { è pari al numero dei lati del } \\
\text { poligono }(n) \text {. }\end{array}$ & $\begin{array}{l}\text { In un poligono angoli esterni } \\
\text { e angoli interni corrispon- } \\
\text { denti sono supplementari. }{ }^{26}\end{array}$ \\
\hline & \multirow[t]{2}{*}{$\begin{array}{l}\text { La somma delle ampiezze } \\
\text { degli angoli interni di un } \\
\text { poligono si ottiene } \\
\text { moltiplicando } 180^{\circ} \text { per il } \\
\text { numero dei triangoli. }\end{array}$} & $\begin{array}{l}\text { La somma delle ampiezze } \\
\text { degli angoli interni dei } \\
\text { triangoli ottenuti dalla } \\
\text { triangolazione è pari a } 180^{\circ} \\
\text { moltiplicato per il numero di } \\
\text { triangoli. }\end{array}$ & $\begin{array}{l}\text { La somma delle ampiezze } \\
\text { degli angoli interni ed } \\
\text { esterni di un poligono è pari } \\
\text { a } 180^{\circ} \text { moltiplicato per il } \\
\text { numero di angoli (interni o } \\
\text { esterni) del poligono. }\end{array}$ \\
\hline & & $\begin{array}{l}\text { La somma delle ampiezze } \\
\text { degli angoli interni di un } \\
\text { poligono si ottiene } \\
\text { sottraendo l'ampiezza di un } \\
\text { angolo giro alla somma delle } \\
\text { ampiezze degli angoli interni } \\
\text { dei triangoli ottenuti dalla } \\
\text { triangolazione. }\end{array}$ & $\begin{array}{l}\text { La somma delle ampiezze } \\
\text { degli angoli interni ed } \\
\text { esterni di un poligono è pari } \\
\text { a } 180^{\circ} \text { moltiplicato per il } \\
\text { numero di angoli (interni o } \\
\text { esterni) del poligono. }\end{array}$ \\
\hline
\end{tabular}

26. In linea di principio, questo risultato potrebbe anche essere trattato precedentemente nel testo, e utilizzato in questo caso come prerequisito. Tuttavia, nell'esempio mostrato (e nelle altre porzioni di libri del corpus in cui si utilizza la strategia /3) esso viene sempre presentato nel momento in cui si affronta la somma delle ampiezze degli angoli interni di un poligono, motivo per cui lo abbiamo inserito tra i risultati matematici. 


\begin{tabular}{|l|l|l|l|}
\hline $\begin{array}{l}\text { Passaggi } \\
\text { aritmetico-algebrici }\end{array}$ & Moltiplicazione: $(n-2) \cdot 180^{\circ}$. & $\begin{array}{l}\text { Moltiplicazione: "angoli interni } \\
\text { dei triangoli }=6 \cdot 180^{\circ} . "\end{array}$ & $\begin{array}{l}\text { Moltiplicazione: } \\
S_{i}+S_{e}=5 \cdot 180^{\circ} .\end{array}$ \\
\hdashline & $\begin{array}{l}\text { Sottrazione: "angoli interni } \\
\text { del poligono } \\
=6 \cdot 180^{\circ}-2 \cdot 180^{\circ} .\end{array}$ & $\begin{array}{l}\text { Sottrazione: } \\
S_{i}=5 \cdot 180^{\circ}-2 \cdot 180^{\circ} .\end{array}$ \\
& & $\begin{array}{l}\text { Proprietà distributiva: } \\
5 \cdot 180^{\circ}-2 \cdot 180^{\circ}=(5-2) \cdot 180^{\circ} .\end{array}$ \\
& & $\begin{array}{l}\text { Sottrazione: } \\
(5-2) \cdot 180^{\circ}=3 \cdot 180^{\circ} .\end{array}$ \\
\hline
\end{tabular}

Tabella 1. Differenze presenti all'interno delle diverse strategie di inventio in termini di prerequisiti, risultati matematici e passaggi aritmetico-algebrici.

In generale, notiamo che la strategia 11 è quella che prevede meno elementi di prerequisiti, risultati matematici nuovi e passaggi aritmetico-algebrici. Dal punto di vista quantitativo si rileva che delle 23 porzioni di testo del sub-corpus, 14 (il 60,9\%) utilizzano la tipologia 11 presente nel caso di studio, 4 (il 17,4\%) la tipologia $12,4(17,4 \%$ ) la tipologia 13 ; in 1 caso, (il 4,3\%) invece, si sceglie di mostrare entrambe le strategie 11 e 12 , anche se queste vengono applicate a un problema specifico e non viene fatta alcuna generalizzazione al poligono generico. ${ }^{27}$ La maggior parte dei libri di testo del corpus utilizza dunque la strategia 11 , che, basandosi su un minor numero di conoscenze da attivare, risulta essere più intuitiva e immediata. Osserviamo però che questa immediatezza potrebbe essere solo apparente, perché si poggia su un fatto geometrico non banale quale è la relazione tra numero di lati del poligono generico e numero di triangoli ottenuti da quel tipo di triangolazione $(n-2)$, relazione che dal lato pedagogico è plausibile interpretare come cognitivamente complessa da accettare. II punto delicato in questa strategia è proprio riuscire a convincere gli allievi della validità di questa relazione. È interessante aggiungere, a queste già di per sé significative differenze di inventio, altre riflessioni sulle diverse realizzazioni argomentative in termini di dispositio ed elocutio rispetto al caso di studio, di cui daremo riscontro nel prossimo paragrafo.

\subsection{La varietà della dispositio ed elocutio per la somma delle ampiezze degli angoli interni di un poligono}

Dai due esempi riportati nel paragrafo precedente si evince come, anche a livello di dispositio ed elocutio, le scelte possono essere molto varie. ${ }^{28}$ In questo paragrafo mostreremo solo gli aspetti più generali, senza entrare negli aspetti più puntuali, perché già da questi si intuisce la grande variabilità di scelte possibili. Nell'esempio in cui si utilizza la strategia I2 (Figura 2), si nota a livello di organizzazione del discorso che il testo è composto dalle fasi dell'esordio e dell'argomentazione, mentre manca un epilogo conclusivo. In questo caso, l'esordio coincide con l'enunciazione della tesi, a cui segue l'argomentazione a sostegno della stessa. L'argomentazione è composta da tre blocchi linguistico-figurali, disposti in modo sequenziale. Nei primi due blocchi viene descritto il procedimento relativo alla costruzione geometrica da realizzare, riferito a un esagono. Significativo è notare l'utilizzo di un linguaggio che, a differenza di quanto accadeva nel caso di studio, tenta di coinvolgere in prima persona il lettore attraverso l'uso di verbi iussivi alla seconda persona singolare ("Disegna", "segna", "congiungi" ecc.). Questo tipo di strategia comunicativa può variare molto a seconda dell'impostazione adottata dal testo (come mostra l'analisi condotta in Sbaragli, Canducci \& Demartini, 2021), e in generale prevedere coinvolgimenti molto diversi del lettore, ad esempio stimolandolo a operare

27. C'è da dire che questo caso rientra all'interno di un libro, in uso nel Canton Ticino, che è particolarmente attento a dare spazio alla dimensione dei problemi matematici da cui far emergere riflessioni e ragionamenti, non solo risultati.

28. Abbiamo scelto di analizzare le scelte di dispositio ed elocutio negli esempi già mostrati in precedenza, evitando di appesantire la trattazione con ulteriori porzioni di libri di testo. Questo da una parte ci consente di andare in profondità rispetto agli esempi presentati, rinunciando d'altra parte a caratterizzare le scelte possibili di dispositio ed elocutio riferite a un'inventio geometrica specifica. 
direttamente congetture sulla base di attività più o meno laboratoriali. Nel terzo blocco vengono invece presentati i passi del ragionamento che conducono alla giustificazione della tesi, rinunciando al coinvolgimento diretto del lettore.

A livello di elocutio multimodale, si nota globalmente la scelta di rappresentare l'esagono tre volte, in seguito alla parte linguistica di ciascuno dei tre blocchi. Notiamo anche che il punto rappresentato all'interno dell'esagono, cruciale per la triangolazione proposta, risulta essere in una posizione centrale della figura, cosa che non solo non sarebbe necessaria ai fini della validità dell'argomento, ma rischia di portare con sé interpretazioni non desiderate, perché un lettore potrebbe pensare che la triangolazione sia valida solo quando il punto interno al poligono è scelto in una posizione privilegiata. Infine, anche in questo caso si è scelto di utilizzare strategie semiotiche di enfatizzazione del testo (il grassetto e la sottolineatura nel primo enunciato).

Si notano varie differenze di dispositio ed elocutio anche con l'esempio riferito alla strategia di inventio 13 (Figura 3). A livello di dispositio, si nota l'assenza di un esordio: il testo procede direttamente presentando l'argomentazione seguita dall'epilogo, nel quale viene presentata la tesi, mostrando dunque un esempio diverso dai due casi precedenti. L'argomentazione può essere suddivisa in diverse parti. La prima è di tipo linguistico, figurale e simbolico: viene introdotto un dato osservativo (significativamente richiamato dal verbo "Osserva") dell'argomentazione, che sta nel riconoscere che ogni angolo interno forma insieme al corrispondente angolo esterno un angolo piatto; inoltre, a differenza di quanto accadeva nel caso di studio, si introducono fin da subito numerosi simboli, utili nelle parti successive dell'argomentazione. Quanto osservato nella prima parte funge da premessa all'affermazione enunciata nella seconda, significativamente introdotta dal connettivo "Quindi". Dopo aver richiamato il prerequisito relativo al fatto che la somma delle ampiezze degli angoli esterni di un poligono è uguale all'ampiezza dell'angolo giro, nella terza parte si propongono passaggi di tipo aritmetico riferiti al caso del pentagono, arrivando a concludere che "La somma degli angoli interni di un pentagono è uguale a 3 angoli piatti, cioè $540^{\circ}$. A questa conclusione segue un'importante affermazione, che mancava tanto nel caso di studio quanto nell'esempio riferito alla strategia 12: "Questo ragionamento può essere fatto per qualsiasi poligono", che rende esplicita la generalizzazione al caso di un poligono generico a partire dall'esempio mostrato. Segue dunque la tesi, espressa dapprima in forma linguistica ("la somma delle ampiezze degli angoli interni di un poligono qualsiasi è uguale a tanti angoli piatti quanti sono i lati meno due"), poi attraverso una sua riformulazione simbolica (" $S_{i}=(n-2) \cdot 180^{\circ}$ "). Osserviamo che tale scelta sembra essere, rispetto a quanto accadeva nel caso di studio, più ordinata e chiara, ma più complessa dal punto di vista della gestione degli aspetti aritmetico-algebrici.

Dal punto di vista dell'elocutio multimodale, anche in questo caso si notano strategie semiotiche utili a enfatizzare alcune parti del discorso (uso del colore nella rappresentazione degli angoli del pentagono, uso del grassetto, uso del colore nel box in cui è racchiusa la prima formulazione della tesi). Rispetto al caso di studio e all'esempio precedente, inoltre, viene fatto un maggiore uso del simbolismo matematico: al di là dei simboli $n, S_{i}$ ed $S_{e}$, utilizzati poi nelle varie parti dell'argomentazione, emerge la scelta, forse esageratamente pedante e non necessaria, di introdurre anche un simbolismo per gli angoli interni ed esterni rappresentati in figura.

Può essere interessante esplicitare che, delle 23 porzioni di testo del corpus, indipendentemente dall'utilizzo delle strategie 11,12 e $13,19\left(I^{\prime} 82,6 \%\right)$ scelgono di proporre la tesi come epilogo del discorso; 1 caso (il 4,3\%) presenta la tesi all'interno dell'argomentazione, facendola seguire da un esempio illustrativo della strategia geometrica adottata; 1 (il 4,3\%), corrispondente all'esempio mostrato in precedenza, pone la tesi come esordio del discorso; infine, 2 casi (l'8,7\%) non esplicitano la tesi: un libro stimola lo studente a ipotizzarla in modo autonomo, I'altro invece si limita a raggiungere la conclusione riferita al caso specifico di un pentagono. Questo è indice di una preferenza generale 
dei libri di testo per la scuola, a cui potrebbe corrisponderne un'altra a livello di pratiche didattiche, di terminare il discorso con una conclusione che è anche la tesi cui si voleva giungere. ${ }^{29} \mathrm{Abbiamo}$ inoltre rilevato che delle 23 porzioni di testo, 9 (il 39,1\%) scelgono di proporre un esordio per introdurre il tema, mentre 14 (il 60,9\%) iniziano direttamente dalla parte argomentativa. Si nota dunque una certa tendenza a entrare direttamente dentro al tema (in medias res, come direbbe uno studioso di retorica), senza preamboli introduttivi, il che potrebbe disorientare il lettore bisognoso di capire il senso di quello che sta per affrontare.

Ovviamente, la variabilità delle scelte di dispositio ed elocutio è presente anche all'interno di ciascuna categoria di inventio, ma in misura meno significativa, motivo per cui abbiamo deciso di non renderne conto in questo contributo. D'altra parte, anche solo analizzando gli aspetti più generali presenti negli esempi mostrati, risulta evidente il livello di eterogeneità che le lenti teoriche da noi adottate riescono a far emergere.

\section{Conclusioni}

Le analisi condotte sulla base del quadro teorico presentato consentono di trarre alcune conclusioni. In primo luogo, ci sembra opportuno rimarcare come non fosse scontata la possibilità di applicare categorie di analisi di tipo retorico, oggi afferenti ai domini delle scienze della comunicazione e delle teorie dell'argomentazione, al caso di libri di testo di matematica. Le analisi condotte hanno mostrato non solo che questo è possibile, ma che riflettere su testi argomentativi di matematica attraverso le lenti proposte permette di mettere in evidenza, e anche in parte di sciogliere, la complessità di questi testi, nei quali si intrecciano dimensioni legate al tipo di argomenti proposti (l'inventio), al modo con il quale si struttura il discorso (la dispositio) e alla forma linguistico-testuale che viene adottata (l'elocutio). In particolare, a livello di inventio, I'esplicitazione delle strutture di ragionamento ha messo in evidenza come esistano diversi modi di trattare la generalizzazione all'interno di argomentazioni non dimostrative: la generalizzazione può essere infatti più o meno esplicitata linguisticamente, e più o meno facilitata dall'uso di esempi come sostegno empirico al passo induttivo. Sempre a livello di inventio, I'esplicitazione dei luoghi ha fatto emergere una serie di meccanismi inferenziali di base di varia natura, che potrebbero non rientrare in un patrimonio già consolidato degli studenti.

A livello di dispositio ed elocutio, I'esplicitazione della struttura organizzativa del testo ha messo in evidenza da un lato le possibilità comunicative offerte dall'utilizzo delle parti del discorso, dall'altro ha consentito di focalizzare l'attenzione su alcune scelte stilistiche che potrebbero risultare problematiche. Su questo ultimo punto in particolare, emergono scelte stilistiche marcate che a volte vanno in contraddizione con il carattere di genericità che richiederebbe la disciplina, e come alcune scelte di enfatizzazione e de-enfatizzazione possano produrre ambiguità di natura semantica e inferenziale. Questo tipo di analisi diventa dunque importante, perché consente - al ricercatore, ma anche all'insegnante che utilizza il libro nella pratica didattica - di guardare al testo con un occhio fine e critico, che può favorire una maggiore consapevolezza di quali siano i dettagli comunicativi con i quali il testo "parla"; questo occhio critico può risultare utile anche per ciò che avviene in classe in termini di oralità. Ad esempio, l'insegnante potrebbe scegliere di adottare argomenti, ordini del discorso o stili comunicativi differenti in base a questioni di carattere didattico, riferite alla disciplina matematica, ma anche contestuali, riferite cioè al particolare uditorio dato dagli studenti della classe: quali strategie argomentative sono più opportune in base alle conoscenze dei miei studenti? È opportuno

29. Notiamo che ciò differisce da quanto accade abitualmente nei testi e nella pratica didattica universitaria, in cui solitamente si preferisce enunciare il teorema e poi la sua dimostrazione. 
presentarne una sola o più di una? Quanti e quali esempi proporre nell'argomentazione che si vuol condurre? Come organizzare il discorso in modo che esso risulti il più efficace possibile dal punto di vista comunicativo? E ancora: come disporre, ad esempio alla lavagna, il testo scritto, le figure e i simboli matematici in modo che sostengano il ragionamento invece di confonderlo? Quali attenzioni linguistiche adottare perché il discorso non presenti oscurità parziali? Quale equilibrio tenere tra il dire troppo, rischiando di annoiare qualcuno, e il dire troppo poco, rischiando di perderne qualcun altro per strada?

Tutte queste domande, alle quali potrebbero esserne aggiunte altre, sono da un lato spunti di riflessione per i docenti, ma anche proficui campi di studio per i ricercatori in didattica della matematica, che potrebbero indagare gli effetti, in termini di comprensione, delle diverse scelte linguistico-comunicative adottabili con gli studenti.

\section{Bibliografia}

Aristotele. (1973). Opere. Laterza.

Austin, J. L. (1987). Come fare cose con le parole. Marietti. (Titolo originale: How to do things with words, pubblicato nel 1962).

Balacheff, N. (2001). Imparare la prova. Pitagora.

Bellucci, C., \& Blotti, A. (2017). Un'esemplificazione dagli Elementi di Euclide. In P. Nanni, E. Rigotti \& C. Wolfsgruber (A cura di), Argomentare per un rapporto ragionevole con la realtà (pp. 209-220). Fondazione per la Sussidiarietà.

Bezemer, J., \& Kress, G. (2010). Changing Text: A Social Semiotic Analysis of Textbooks. Designs for Learning, $3(1-2), 10-29$.

Canducci, M. (2019). II rapporto testo-figure nei libri di testo di matematica: il caso dei poligoni analizzato in ottica multimodale. In B. D'Amore \& S. Sbaragli (A cura di), Didattica della matematica e professionalità docente, Atti del XXXIII convegno di Castel San Pietro Terme (pp. 107-108). Pitagora.

Canducci, M. (2020). L'incoerenza delle scelte di numero nei libri di testo di geometria. In B. D'Amore \& S. Sbaragli (A cura di), Didattica della matematica, disciplina scientifica per una scuola efficace, Atti del XXXIV convegno di Castel San Pietro Terme (pp. 69-70). Pitagora.

Canducci, M., Demartini, S., Franchini, E., \& Sbaragli, S. (2019a). La definizione nei testi scolastici: dall'analisi alla didattica. In B. Di Paola (A cura di), Pratiche d'aula e ricerca didattica: nuove e vecchie sfide di insegnamentolapprendimento matematico per una scuola competente e inclusiva (pp. 47-48). "Quaderni di Ricerca in Didattica", n. 2 Numero speciale n. 5, 2019. G.R.I.M. http://math.unipa.it/ grim/quaderno2 suppl 52019. $\underline{\mathrm{pdf}}$

Canducci, M., Demartini, S., Franchini, E., \& Sbaragli, S. (2019b). Analisi di manuali scolastici di matematica dal punto di vista linguistico e disciplinare. In B. Di Paola (A cura di), Pratiche d'aula e ricerca didattica: nuove e vecchie sfide di insegnamento/apprendimento matematico per una scuola competente e inclusiva (pp. 43-44). "Quaderni di Ricerca in Didattica", n. 2 Numero speciale n.5, 2019. G.R.I.M. http://math.unipa. it/ grim/quaderno2 suppl 5 2019.pdf

Canducci, M., Demartini, S., \& Sbaragli, S. (2021). Plurale o singolare? Disomogeneità linguistica di numero nei manuali di matematica della scuola primaria e secondaria di primo grado italiani. Italiano a scuola, 3(1), 99-132. https://doi.org/10.6092/issn.2704-8128/12935 
Canducci, M., Rocci, A., \& Sbaragli, S. (2021). The influence of multimodal textualization in the conversion of semiotic representations in Italian primary school geometry textbooks. Multimodal Communication, 10(2), 157-174. https://doi.org/10.1515/mc-2020-0015

Cattani, A. (1994). Forme dell'argomentare. I/ ragionamento tra logica e retorica. Edizioni GB.

Cicerone. (1994). Dell'oratore. BUR.

Cornificio. (1993). Rhetorica ad Herennium. Pàtron.

Demartini, S., Fornara, S., \& Sbaragli, S. (2020). Se la sintesi diventa un problema. Alcune caratteristiche del linguaggio specialistico della matematica in prospettiva didattica. In J. Visconti, M. Manfredini \& L. Coveri (A cura di), Linguaggi settoriali e specialistici: sincronia, diacronia, traduzione, variazione. Atti del XV Congresso Internazionale SILFI, Genova, 28-30 maggio 2018 (pp. 487-494). Cesati.

Demartini, S., \& Sbaragli, S. (2019). Le parole che "ingannano". La componente lessicale nell'insegnamento e nell'apprendimento della matematica. In B. Di Paola (A cura di), Pratiche d'aula e ricerca didattica: nuove e vecchie sfide di insegnamento/apprendimento matematico per una scuola competente e inclusiva (pp. 19-25). "Quaderni di Ricerca in Didattica", n. 2 Numero speciale n.5, 2019. G.R.I.M. http://math.unipa. it/grim/quaderno2 suppl 5 2019.pdf

Demartini, S., Sbaragli, S., \& Ferrari, A. (2020). L'architettura del testo scolastico di matematica per la scuola primaria e secondaria di primo grado, Italiano LinguaDue, 12(2), 160-180. www.italianolinguadue.unimi.it

Duval, R. (1993). Registres de représentations sémiotique et fonctionnement cognitif de la pensée. Annales de Didactique et de Sciences Cognitives, 5, 57-65.

Ferrari, P. L. (2021). Educazione matematica, lingua, linguaggi. Costruire, condividere e comunicare matematica in classe. Utet università.

Kjeldsen, J. E. (2015). The study of visual and multimodal argumentation. Argumentation, 29(2), 115-132.

Lausberg, H. (1969). Elementi di retorica. II Mulino. (Titolo originale: Elemente der literarischen Rhetorik pubblicato nel 1949).

Lavinio, C. (2004). Comunicazione e linguaggi disciplinari. Per un'educazione linguistica trasversale. Carocci.

Leal, F. (2016). Review of: Frans H. van Eemeren (2015): Reasonableness and Effectiveness in Argumentative Discourse: Fifty Contributions to the Development of Pragma-Dialectics, Argumentation, 30, 527-532. https:// doi.org/10.1007/s10503-016-9393-7

Maggi, A. (2017). Funzioni dell'inferenza nel ragionare matematico. In P. Nanni, E. Rigotti \& C. Wolfsgruber (A cura di), Argomentare per un rapporto ragionevole con la realtà (pp. 221-237). Fondazione per la Sussidiarietà.

Mortara Garavelli, B. (2020). Manuale di retorica. Bompiani (edizione originale pubblicata nel 1988).

Mercier, H., \& Sperber, D. (2017). The enigma of reason. Harvard University Press.

Novaes, C. D. (2020). The dialogical roots of deduction: Historical, cognitive, and philosophical perspectives on reasoning. Cambridge University Press.

Perelman, C., \& Olbrechts-Tyteca, L. (2013). Trattato dell'argomentazione. La nuova retorica. Giulio Einaudi editore (Titolo originale: La nouvelle rhétorique. Traité de l'Argumentation pubblicato nel 1958).

Piazza, F. (2015). La Retorica di Aristotele. Introduzione alla lettura. Carocci. 
Pollaroli, C., \& Rocci, A. (2015). The argumentative relevance of pictorial and multimodal metaphor in advertising. Journal of argumentation in context, 4(2), 158-199.

Rigotti, E., \& Greco, S. (2009). Argumentation as an Object of Interest and as a Social and Cultural Resource. In N. M. Mirza \& A.-N. Perret-Clermont (Eds.), Argumentation and Education: Theoretical Foundations and Practices (pp. 1-61). Springer.

Rigotti, E., \& Greco, S. (2019). Inference in Argumentation: A Topics-Based Approach to Argument Schemes. Springer.

Rocci, A. (1996). Valori comunicativi della posizione dell'aggettivo in italiano. Vita e pensiero.

Rocci, A. (2017). Ragionevolezza dell'impegno persuasivo. In P. Nanni, E. Rigotti \& C. Wolfsgruber (A cura di), Argomentare per un rapporto ragionevole con la realtà (pp. 88-120). Fondazione per la Sussidiarietà.

Rocci, A., \& Pollaroli, C. (2018). Introduction: Multimodality in argumentation. Semiotica, 2018(220), 1-17.

Sbaragli, S., Canducci, M., \& Demartini, S. (2021). Le modalità logico-argomentative nei testi scolastici di geometria della scuola elementare e media in lingua italiana. Didattica della matematica. Dalla ricerca alle pratiche d'aula, 9, 44-71. https://doi.org/10.33683/ddm.21.9.3

Sbaragli, S., \& Demartini, S. (A cura di). (2021). Italmatica. Lingua e strutture dei testi scolastici di matematica. Dedalo.

van Eemeren, F. H. (2013). In what sense do modern argumentation theories relate to Aristotle? The case of pragma-dialectics. Argumentation, 27(1), 49-70.

van Eemeren, F. H. (2015). Reasonableness and Effectiveness in Argumentative Discourse: Fifty Contributions to the Development of Pragma-Dialectics. Springer.

Walton, D., Reed, C., \& Macagno, F. (2008). Argumentation schemes. Cambridge University Press.

Whately, R. (1963). Elements of rhetoric. Southern Illinois University Press (edizione originale pubblicata nel 1828). 